\title{
Fission half-lives of superheavy nuclei in a microscopic approach
}

\author{
M. Warda ${ }^{*}$ \\ Katedra Fizyki Teoretycznej, Uniwersytet Marii Curie-Skłodowskiej, ul. Radziszewskiego 10, 20-031 Lublin, Poland \\ J. L. Egido ${ }^{\dagger}$ \\ Departamento de Física Teórica, Universidad Autónoma de Madrid, 28049 Madrid, Spain \\ (Received 26 April 2012; published 19 July 2012)
}

\begin{abstract}
A systematic study of 160 heavy and superheavy nuclei is performed in the Hartree-Fock-Bogoliubov (HFB) approach with the finite-range and density-dependent Gogny force with the D1S parameter set. We show calculations in several approximations: with axially symmetric and reflection-symmetric wave functions, with axially symmetric and non-reflection-symmetric wave functions, and finally with some representative triaxial wave functions. Relevant properties of the ground state and along the fission path are thoroughly analyzed. Fission barriers, $Q_{\alpha}$ factors, and lifetimes with respect to fission and $\alpha$ decay as well as other observables are discussed. Larger configuration spaces and more general HFB wave functions as compared to previous studies provide a very good agreement with the experimental data.
\end{abstract}

DOI: 10.1103/PhysRevC.86.014322

PACS number(s): 21.60.Jz, 21.10.Dr, 25.85.Ca, 27.90.+b

\section{INTRODUCTION}

The stability and structure of nuclei at the upper end of the nuclear chart is a hot topic in contemporary nuclear physics. It is a challenging task to answer which heavy nuclides may exist and what properties they may have. Therefore strong efforts have been made in the experimental developments as well as in the theoretical description of superheavy elements (SHEs).

In the last decades huge progress in the synthesis of new elements has been achieved in world-leading laboratories such as the GSI, Darmstadt [1-8], the Joint Institute for Nuclear Research (JINR), Dubna [9-14], and Rikagaku Kenkyusho (Institute of Physical and Chemical Research, Japan; RIKEN), Tokyo [15-18]. The first SHEs with $Z \leqslant 113$ and $N \leqslant 165$ were produced in cold fusion reactions. These experiments, involving neutron-rich projectiles and spherical targets $\left({ }^{208} \mathrm{~Pb}\right.$ or ${ }^{209} \mathrm{Bi}$ ), produced weakly excited compound nuclei, which cooled by the emission of only one or two neutrons. Further experimental progress in the synthesis of the heaviest elements was achieved by hot fusion reactions in which targets of deformed actinides were bombarded with the doubly magic nucleus ${ }^{48} \mathrm{Ca}$. The compound nucleus created in this way was more excited and three or more neutrons were emitted. These reactions succeeded in the synthesis of new elements up to $Z=118$ and $N=176$ [10]. The first observation of element 117 has been made possible lately [13] using a radioactive ${ }^{249} \mathrm{Bk}$ target. New possibilities for the synthesis of new isotopes will be opened up in heavy-ion collisions with radioactive ion beams $[19,20]$. Nowadays, many other laboratories are involved in the exploration of SHEs. Thus the experimental groups from Berkeley [21], the Grand Accélérateur National d'Ions Lourds (GANIL) [19], Livermore [11,22], Jyväskylä [23,24], and Oak Ridge [14] are working in this direction. They would bring in the near future further information on

\footnotetext{
*warda@kft.umcs.lublin.pl

†j.luis.egido@uam.es
}

the stability and properties of the SHEs and an independent verification of the existing data.

In a parallel way to the experimental efforts, the properties of the SHEs have been also investigated in various nuclear models. A proper description of trans-fermium nuclei is a great challenge for any theoretical model. Usually, the parameters of the theories on atomic nuclei are adjusted to the stable isotopes and then extrapolated to the region of heavier systems. Therefore many tries in different theoretical approaches are performed to foresee the stability and the structure of the heaviest nuclei. A detailed review of the theoretical analysis of SHEs can be found in Ref. [25]. The first theoretical investigations on the stability of heavy nuclei were made in the 1960s. It was noticed that shell effects could stabilize nuclei heavier than those known at that time [26,27]. Calculations made in the macroscopic-microscopic model with a Strutinsky shell correction predicted the "island of stability." Large values of shell energies were obtained at $Z=108, N=162$ for prolate-deformed nuclei and $Z=114, N=184$ for spherical ones [26,28]. In the last decades many calculations have been made, providing more and more precise predictions. The fission barriers and the ground-state properties were calculated using macroscopic-microscopic methods over a large range of deformation parameters and nuclear shapes, including reflection- and axial-symmetry breaking [29-40].

Self-consistent methods also provided many results on fission barriers and half-lives. Important results have been obtained in the relativistic mean-field (RMF) [40-46] approach, the Hartree-Fock (HF) approach with Skyrme forces $[41,42,47-51]$, and the Hartree Fock-Bogoliubov (HFB) theory with Gogny forces [52-54]. The first calculations of fission barriers were performed in the axial- and reflection-symmetric regime but later all relevant deformations were considered in the minimization of the energy.

It is well known that the liquid drop model does not predict a fission barrier in the heaviest nuclei and that the stability of the trans-fermium nuclei is achieved by the shell effects. The selfconsistent quantum mechanical methods (RMF, HF, and HFB) 
as microscopic theories are the perfect tools for the analysis of SHEs. Moreover, in the self-consistent calculations all possible shapes of a nucleus are considered in the minimization process. In contrast, most of the macroscopic-microscopic models are restricted to some predefined classes of deformations and only "optimal shapes" [55] allow us to obtain any configuration of a nucleus. Therefore they are very suitable to describe large deformations of nuclei around the scission point. It is well known that pairing correlations play an important role along the fission path [56]. Since in the HFB theory the particle-hole and the particle-particle matrix elements are treated on the same footing, the proper consideration of pairing along the whole fission path is guaranteed. This method has been successfully applied in many aspects of low-energy nuclear physics, in particular in the description of fission barriers of heavy nuclei $[52,53,57]$. Another theoretical quest was to discover a semiempirical formula describing $\alpha$-emission half-lives $[58,59]$. These investigations are very important as $\alpha$ radioactivity is the dominant decay channel in many SHEs.

The purpose of this article is to perform a systematic study of SHEs with respect to their stability and ground-state properties in the framework of the HFB theory with the density-dependent finite-range Gogny force and the D1S parametrization. In our analysis we include the region of the well-known fermium (Fm, $Z=100$ ) and nobelium (No, $Z=$ 102) elements to compare our predictions with the available experimental data. We show results for the heavier eveneven nuclei rutherfordium ( $\mathrm{Rf}, Z=104)$, seaborgium $(\mathrm{Sg}$, $Z=106$ ), hassium (Hs, $Z=108$ ), darmstadtium (Ds, $Z=$ $110)$, and copernicium $(\mathrm{Cn}, Z=112$, which was named two years ago [60]). Heavier elements with $Z=114-124$, without having been given names so far, are also considered. We limit our study to $N \leqslant 190$ isotopes. These nuclei are nowadays in the mainstream of interest of experiments with SHEs.

A large amount of information on nuclear structure and stability can be obtained from properties of the ground states of the SHEs. Consequently, we start our investigation with the description of the ground-state characteristics. Deformations, pairing energies, and two-nucleon separation energies are analyzed and collated with the single-particle energy level scheme. The ground-state energies can be used to evaluate the $Q_{\alpha}$ values which are necessary to calculate the probability of $\alpha$ emission-one of the dominant decay modes in SHEs. A competing process to $\alpha$ decay is spontaneous fission. To analyze this mode we determine the fission barriers for all mentioned SHEs as a function of the quadrupole moment $Q_{2}$. The calculations were performed in an axial basis, although we are aware of nonaxial effects on the height of the barrier and we discuss them in a few selected cases. The impact of the octupole deformation on the potential energy along the fission path is crucial in the determination of fission barriers of SHEs. This is taken into account by allowing non-reflection-symmetrical shapes. The calculations were performed in a large deformed harmonic oscillator basis, paying special attention to the proper optimization of the oscillator lengths and to the convergence of the calculations with the size of the basis. Next, using the WKB approximation, we calculate the fission half-lives. Finally, the comparison of the half-lives for $\alpha$ decay and spontaneous fission allows us to predict the stability of the heaviest nuclei.
This article is organized as follows. In Sec. II we briefly describe the constrained Hartree-Fock-Bogoliubov calculations. The description of the ground-state properties of SHEs is shown in Sec. III, fission barriers are discussed in Sec. IV, and half-lives of SHEs are discussed in Sec. V. Finally, Sec. VI contains a summary and some concluding remarks.

\section{THEORETICAL MODEL}

In our research we will apply the self consistent Hartree-Fock-Bogoliubov theory with the finite-range densitydependent Gogny force. In the numerical applications we use the D1S $[61,62]$ parametrization of the Gogny interaction. The D1S parameters were adjusted [61] to give a better surface energy term (which is crucial for a proper description of the fission phenomenon). The choice of the Gogny force with the D1S parametrization is based on the fact that whenever this interaction has been used to describe low-energy nuclear structure phenomena an at least reasonable agreement with experiment has been obtained. This degree of agreement has been obtained for calculations at both the mean-field level and beyond [63-79].

\section{A. Details of self-consistent HFB calculations}

In the microscopic HFB calculations we have used the computer code presented in Ref. [80] (see also [57] where special attention was paid to an accurate computation of the matrix elements of the Gogny interaction for very large basis like the one used in this paper). The self-consistent equations have been solved by expanding the quasiparticle creation and annihilation operators on finite bases of axially symmetric deformed harmonic oscillator (HO) eigenfunctions. The size of the bases used depends upon two parameters, $N_{0}$ and $q$, which are related to the allowed range of the $\mathrm{HO}$ quantum numbers through the relation

$$
\frac{1}{q} n_{z}+\left(2 n_{\perp}+|m|\right) \leqslant N_{0} .
$$

Along the perpendicular direction we take $N_{0}$ shells (i.e., $\left.2 n_{\perp}+|m|=0, \ldots, N_{0}\right)$ and along the $z$ direction we include up to $q N_{0}$ shells depending on the value of $2 n_{\perp}+|m|$. In the present study we have used $q=1.5$, a value which is suited for the elongated shapes along the $z$ direction typical of the fission process, and $N_{0}=15$. Other parameters characterizing the $\mathrm{HO}$ bases are the oscillator lengths $b_{\perp}$ and $b_{z}$. These two quantities have been determined, for each calculated wave function, so as to minimize the HFB energy. In order to study the different paths to fission, in our calculations we have used as constraints the axial quadrupole $\left(Q_{2}\right)$ and octupole $\left(Q_{3}\right)$ moments, with $\hat{Q}_{\lambda}=r^{\lambda} P_{\lambda}(\cos \theta)$. Higher multipolarities are adjusted in the self-consistent process to minimize the energy. To study the impact of triaxiality effects we have also carried out calculations for a few nuclei where the axial symmetry requirement was released but the left-right symmetry was imposed. For the deformation parameters $\beta_{n}$, since most of the studies on these parameters have been done in the macroscopic-microscopic models we will use 
a definition in the spirit of these models. The deformation parameters $\beta_{n}$ entering in $R(\theta)=R_{0}\left[1+\sum_{n=2}^{10} \beta_{n} P_{n}(\cos \theta)\right]$ are determined in such a way that the multipole moments ( $\left.Q_{\lambda}, \lambda=2-10\right)$ calculated with $R(\theta)$ and the HFB selfconsistent ones coincide.

In the calculations the Coulomb exchange energy has been treated in the Slater approximation $[81,82]$. Additionally, the Coulomb and the spin-orbit contributions to the pairing field have been neglected. Finally, the two-body kinetic energy correction (2b-KEC) is not included in the variation process because, for heavy nuclei, it remains almost constant for most of the physical configurations. As this term was included in the fitting of the force, we have to include its contribution at the end of the variational process in order to obtain reasonable binding energies. For a quantitative discussion of the terms relevant to fission see Fig. 2 of Ref. [57]. For the quantitative discussion of all terms in the general case see Ref. [82].

We have also subtracted from the HFB energy the rotational energy corrections (RECs) stemming from the restoration of rotational symmetry. This correction has a considerable influence on the energy landscape (and therefore on the height of the fission barriers) as it is somehow proportional to the degree of symmetry breaking and therefore proportional to the quadrupole moment. A full calculation of the REC would imply an angular momentum projection [72,83-85] which is only feasible for light nuclei. In order to estimate the REC we have followed the usual recipe [86] of subtracting from the HFB energy the quantity $\left\langle\Delta \vec{J}^{2}\right\rangle /\left(2 \mathcal{J}_{Y}\right)$, where $\left\langle\Delta \vec{J}^{2}\right\rangle$ is the fluctuation associated with the angular momentum operators in the HFB wave function and $\mathcal{J}_{Y}$ is the YoccoZ moment of inertia [86]. This moment of inertia has been computed using the "cranking" approximation in which the full linear response matrix appearing in its expression is replaced by the zero-order approximation. The effect of the cranking approximation in the Yoccoz moment of inertia was analyzed with the Gogny interaction for heavy nuclei in [87] by comparing it with the one extracted from an angular-momentum-projected calculation (see also [84] for a comparison in light nuclei). The conclusion is that the exact REC is a factor of 0.7 smaller than the one computed with the cranking approximation to the Yoccoz moment of inertia for strongly deformed configurations (and a similar behavior has been observed for the Thouless-Valatin moment of inertia in [88]). We have taken this phenomenological factor into account in our calculation of the REC.

\section{B. Evaluation of lifetimes}

The evaluation of the spontaneous fission half-life is carried out in the standard WKB framework where $T_{\text {sf }}$ is given (in seconds) by [89]

$$
T_{\text {sf }}=2.86 \times 10^{-21}[1+\exp (2 S)] .
$$

In this expression $S$ is the action along the $Q_{2}$-constrained path, which is given by

$$
S=\int_{a}^{b} d Q_{2} \sqrt{2 B\left(Q_{2}\right)\left(V\left(Q_{2}\right)-E_{0}\right)}
$$

$a$ corresponds to the $Q_{2}$ value of the ground state and $b$ to the $Q_{2}$ value where the potential energy equals that of the ground state. For the collective quadrupole inertia $B\left(Q_{2}\right)$ we have used the adiabatic time-dependent HFB (ATDHFB) expression computed again in the cranking approximation and given by [90]

$$
B_{\text {ATDHFB }}\left(Q_{2}\right)=\frac{M_{-3}\left(Q_{2}\right)}{M_{-1}^{2}\left(Q_{2}\right)},
$$

with

$$
M_{-n}\left(Q_{2}\right)=\sum_{\mu \nu} \frac{\left|Q_{\mu \nu}^{20}\right|^{2}}{\left(E_{\mu}+E_{\nu}\right)^{n}} .
$$

Here $Q_{\mu \nu}^{20}$ is the two-particle-zero-hole component of the quadrupole operator $\hat{Q}_{2}$ in the quasiparticle representation [86] and $E_{\mu}$ are the quasiparticle energies obtained in the solution of the HFB equation.

In the expression for the action the collective potential $V\left(Q_{2}\right)$ is given by the HFB energy (with the two-body kinetic energy and rotational energy corrections) minus the zero-point-energy (ZPE) correction $\epsilon_{0}\left(Q_{2}\right)$ associated with the quadrupole motion. This ZPE correction is given by

$$
\epsilon_{0}\left(Q_{2}\right)=\frac{1}{2} G\left(Q_{2}\right) B_{\mathrm{ATDHFB}}^{-1}\left(Q_{2}\right),
$$

where

$$
G\left(Q_{2}\right)=\frac{M_{-2}\left(Q_{2}\right)}{2 M_{-1}^{2}\left(Q_{2}\right)} .
$$

Finally, in the expression for the action an additional parameter $E_{0}$ is introduced. This parameter can be taken as the HFB energy of the (metastable) ground state. However, it is argued that in a quantal treatment of the problem the ground-state energy is given by the HFB energy plus the zero-point energy associated with the collective motion. To account for this fact, the usual recipe [91] is to add an estimation of the zero-point energy to the HFB energy in order to obtain $E_{0}$. In our calculations we have taken a zero-point energy of $0.5 \mathrm{MeV}$ for all the isotopes considered.

In some SHEs around $N=176$ a weakly oblate-deformed ground state can be found. The fission path in these nuclei obviously does not go along axial-symmetric shapes through the spherical configuration. Such a nucleus rather takes triaxial shapes to reach the prolate saddle point with an absolute value similar to that of the quadrupole moment. As the energies on the triaxial part of the fission barrier are very small in comparison with the saddle point [92] they will contribute insignificantly to the action integral. Therefore we will neglect them in the calculation of half-lives.

To calculate the $\alpha$-decay half-lives we use the phenomenological formula of Viola and Seaborg [93],

$$
\log T_{\alpha}[\mathrm{yr}]=(a Z+b)\left(Q_{\alpha}\right)^{-1 / 2}+(c Z+d)-7.5,
$$

with $Z$ the atomic number of the parent nucleus. The $Q$ factor of the decay, $Q_{\alpha}$, is obtained from the calculated groundstate binding energies with the experimental value $E(2,2)=$ -28.295674 MeV [94]:

$$
Q_{\alpha}(Z, N)=E(Z, N)-E(Z-2, N-2)-E(2,2) .
$$


The constants in Eq. (7) are $a=1.66175, b=-8.5166, c=$ -0.20228 , and $d=-33.9069$ (taken from [95]).

\section{GROUND-STATE PROPERTIES}

In Fig. 1 we present an overview of all the nuclei covered in the present work. In the calculations we limit ourselves to eveneven isotopes. The chosen region ranges from the heaviest trans-actinides, well known from numerous experiments, up to beyond the neutron magic shell number $N=184$ predicted by many theoretical models. The upper limit is provided by the vanishing of the two-proton separation energy. The latter approximates the proton drip line, which is determined by the one-proton separation energy with some correction due to the influence of the centrifugal barrier [96]. The region of neutron-rich isotopes beyond the $\beta$-stability line (indicated in Fig. 1 by black squares) has been omitted as it is out of reach for the current experimental methods and cannot be produced in heavy-ion collisions. As can be seen in Fig. 1 the experimentally known SHEs are located in the center of the investigated region. Since we consider the ground-state properties as especially relevant for the understanding of the underlying physics we present these properties first while the following section is entirely devoted to the study of the fission barriers.

The theoretical approach discussed in the previous section has been applied to perform a systematic study of the properties of 160 superheavy nuclei in the region $150 \leqslant N \leqslant 190$, $100 \leqslant Z \leqslant 126$. Earlier calculations in this region have been performed in the microscopic-macroscopic approach $[32,33,39]$, in the HF plus BCS approach with the Skyrme force in the particle-hole channel and a monopole pairing force in the particle-particle channel [41,42,47-51], and in the full HFB approach by $[52,53,97]$ with the Gogny force. The calculations by Berger et al. are rather similar to ours in the basic aspects. However, in our work the configuration space is larger, more appropriate for fission, and we allow more general HFB wave functions (with more simultaneously broken symmetries). Furthermore, our study is rather detailed and systematic.

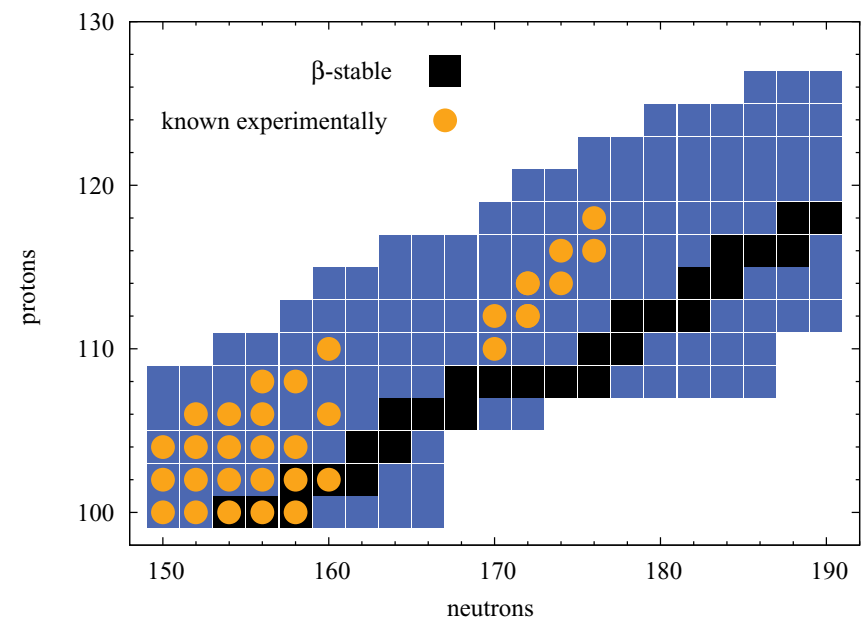

FIG. 1. (Color online) Isotopes considered in this work.

\section{A. Single-particle energies}

To gain insight into the relevant physics of the SHEs we display in Fig. 2 the single-particle energies for the nucleus ${ }^{270} \mathrm{Hs}$ in reflection-symmetric configurations as a function of the quadrupole deformation $\beta_{2}$ and the quadrupole moment $Q_{2}$. We have chosen this nucleus as a representative of the whole region for the following reasons. Since it is in the center of the area of the calculated nuclei its single-particle energy (s.p.e.) spectrum is characteristic for all SHEs; furthermore, its proton and neutron pairing energies are small and the extraction of the s.p.e. values from the HFB calculations is more reliable. The s.p.e.'s are obtained as usual: After the HFB equations are solved the one-body HF Hamiltonian is diagonalized in that basis. For the neutron system we find large spherical gaps at neutron numbers of 164,184 , and 228 , prolate gaps at 162, and oblate gaps at 172 and 178. In nuclei with neutron number close to 160 the spherical minimum at 164 is overwhelmed by the prolate one at 162 . For protons there are several gaps. The more relevant are the spherical ones for $Z$ values of 92,114 , and 126, the spherical-oblate one at 120, and the prolate ones at 104 and 108. As we will see below many nuclear properties can be understood just by looking at these s.p.e.'s.

\section{B. Deformations}

Let us now discuss some ground-state properties starting with the deformation parameters $\beta_{n}$. The deformation parameters $\beta_{2}$ of the calculated SHEs are given in the third column of Table I and they are visualized in Fig. 3(a). The quadrupole deformation parameter $\beta_{2}$ barely depends on the proton number $Z$ and decreases slowly with increasing neutron number. For all $Z$ values the nuclei with $N<170$ have a prolate deformation with $\beta_{2}>0.25$. In the neutron number region $170 \leqslant N \leqslant 182$, the potential energy surfaces (PESs) $[54,98]$ of these nuclei present coexisting prolate and oblate minima. The quoted values for those nuclei are the ones corresponding to the deeper minimum. The prolate minimum is deeper in lighter nuclei with $N \leqslant 174$ and $Z \leqslant 118$, while the oblate one is deeper in the heavier ones. With increasing neutron number the depth of the prolate well becomes smaller compared to the oblate one with the oblate minimum becoming the lowest one around $N=174$ (see also Fig. 10). At the same time the absolute value of the quadrupole deformation parameter of the ground state decreases from $\left|\beta_{2}\right|=0.2$ for $N=172$ to $\left|\beta_{2}\right|=0.05$ for $N=182$. The barrier between the two wells diminishes and finally disappears at $N=184$, where the nuclei become spherical. We also observe in this region a weak $Z$ dependency. For isotopes with $N>184$ small prolate deformation can be observed in the ground state. As compared with other calculations [99-102] of SHEs our $\beta_{2}$ values are somewhat larger but this may be connected to the slightly different methods of computation of the deformation parameters $\beta_{n}$. In the presence of coexisting minima, one must be aware that when the restriction to axially symmetric shapes is released one of the two minima may not be a true minimum, but rather a saddle point [92]. A clear example is the case of the heavier Ds isotopes. In 

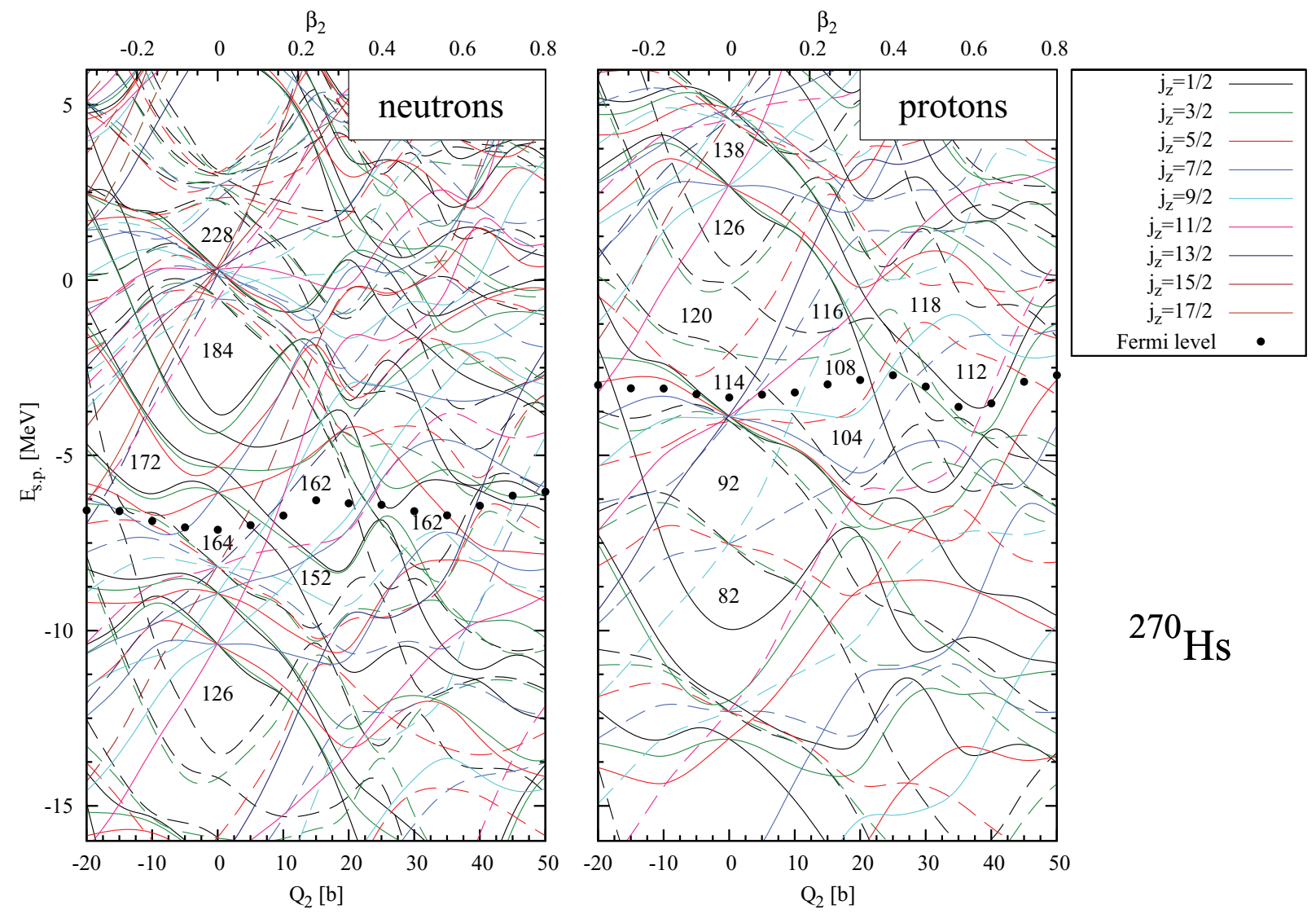

FIG. 2. (Color online) Single-particle energies of the nucleus ${ }^{270} \mathrm{Hs}$ as a function of the deformation parameter $\beta_{2}$ (top $x$ axis) and the quadrupole moment $Q_{2}$ (bottom $x$ axis). Continuous and dashed lines represent positive- and negative-parity states, respectively, and the bullets indicate the Fermi level.

Fig. 10 one observes two minima (prolate and oblate) around $\pm 5 \mathrm{~b}$, but looking at triaxial calculations one finds that the prolate minimum is a saddle point. The gross behavior of the deformation parameter can be qualitatively understood by looking at Fig. 2. Here we observe that shell effects favor strong prolate deformations for the nuclei with $150 \leqslant N \leqslant 162$ and $100 \leqslant Z \leqslant 108$, i.e., deformation-driving (down-sloping) levels are being populated and spherical-driving (up-sloping) levels are being depopulated. In the region $164 \leqslant N \leqslant 170$ and $108 \leqslant Z \leqslant 116$ neutron shell effects favor smaller prolate deformations while in the proton system small prolate and spherical shapes are favored. Around $N=172$ the oblate shapes are favored while larger $N$ values prefer smaller oblaticity. This effect is reinforced by the soft oblate proton gap at $Z=120$. The spherical shape is the privileged one for neutron shell structure at $N=184$. This coincides with the subsequent proton spherical gaps at $Z=114, Z=120$, and $Z=126$, which cover most of the $N=184$ isotones considered here.

In columns 4 to 7 of Table I as well as in Figs. 3(b)-3(d) we present the deformation parameters of higher multipolarities. Most of the nuclei are reflection symmetric in the ground states and only for nuclei with $N>184$ do we obtain octupoledeformed ground states independent of the proton number. Möller et al. [99] also found nonzero $\beta_{3}$ deformations for the
SHEs in this region. The odd multipolarities $\beta_{5}, \beta_{7}$, and $\beta_{9}$, not shown here, are different from zero only for those nuclei whose ground state is octupole deformed; however, the numerical values are very small (e.g., $\beta_{5}$ values are less than 0.009 ). For the even deformations higher than two [see Figs. 3(c) and $3(d)]$, they are different from zero only for nuclei with $\beta_{2} \neq 0$, i.e., for all nuclei with the exception of the few isotopes around $N=186$. As we can observe there is a smaller dependence with $Z$ than with $N$. Nuclei with $N \approx 150$ have small negative hexadecapole deformations. For increasing $N,\left|\beta_{4}\right|$ increases up to $N=168$ where it reaches the largest absolute value. A large negative hexadecapole moment together with a large positive quadrupole moment produces barrel-like shapes in the ground state of nuclei in this region. From here on $\left|\beta_{4}\right|$ decreases rather smoothly up to $N=176$, where it sharply decreases to very small deformations and zero values at the largest neutron number analyzed. This sharp decrease is associated with the prolate-oblate shape transition that takes place at this neutron number. For $N$ values $150 \leqslant N \leqslant 176$, i.e., the prolate part, there is a clear tendency with $Z$ for a given $N$ : with increasing proton number $\left|\beta_{4}\right|$ gets larger. For the oblate-spherical part, there is almost no dependence with $Z$. The behavior of the deformation parameter $\beta_{6}$ is quite different from that of $\beta_{4}$. For $150 \leqslant N \leqslant 160$ we obtain negative values decreasing in absolute value as $N$ increases. Around 
TABLE I. Selected properties of superheavy nuclei: ground-state deformation parameters, pairing energies, $Q_{\alpha}$, two-nucleon separation energies, and half-lives for $\alpha$ emission and spontaneous fission calculated in HFB theory. The energies are given in MeV and half-lives are in seconds.

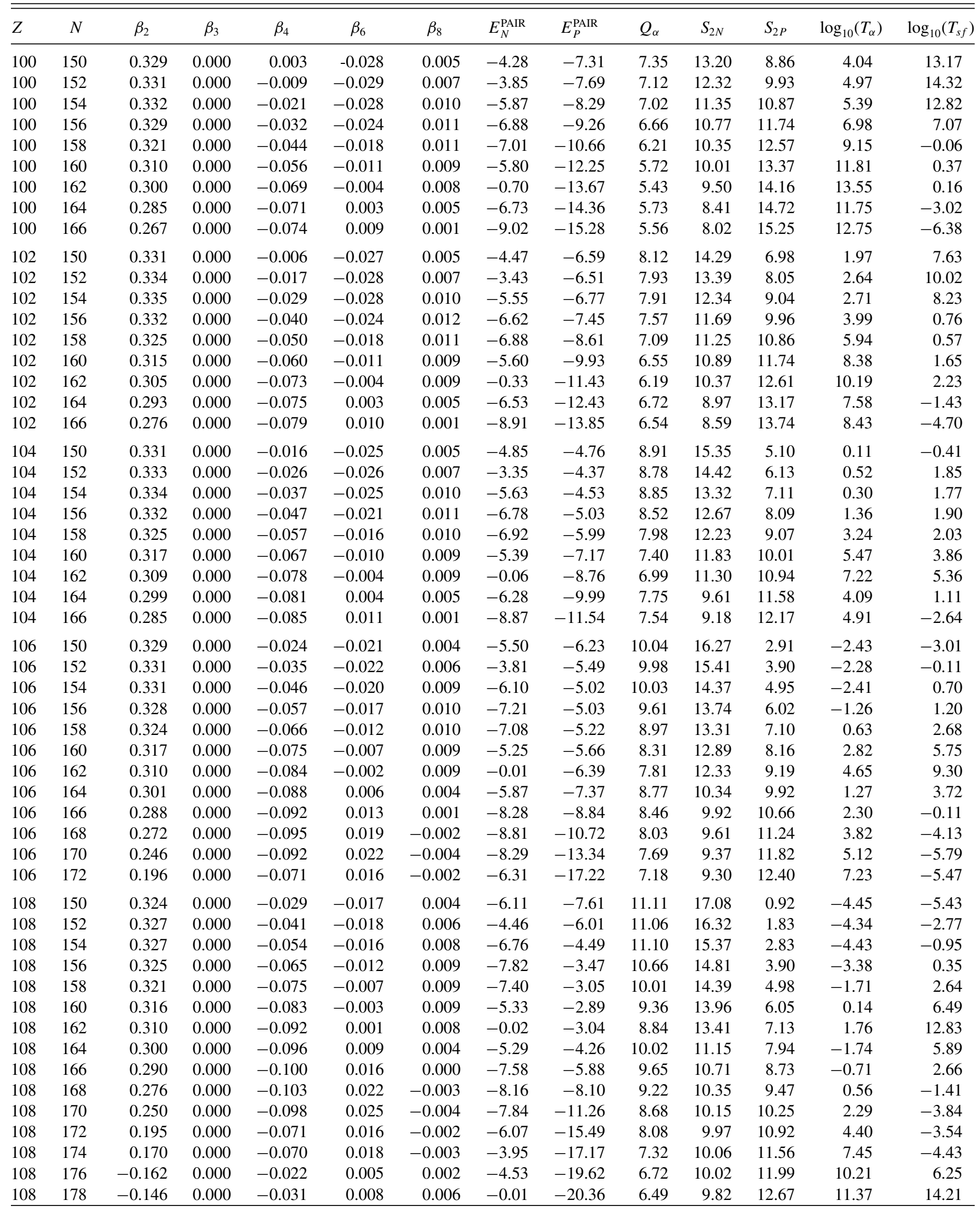


TABLE I. (Continued.)

\begin{tabular}{|c|c|c|c|c|c|c|c|c|c|c|c|c|c|}
\hline$Z$ & $N$ & $\beta_{2}$ & $\beta_{3}$ & $\beta_{4}$ & $\beta_{6}$ & $\beta_{8}$ & $E_{N}^{\mathrm{PAIR}}$ & $E_{P}^{\mathrm{PAIR}}$ & $Q_{\alpha}$ & $S_{2 N}$ & $S_{2 P}$ & $\log _{10}\left(T_{\alpha}\right)$ & $\log _{10}\left(T_{s f}\right)$ \\
\hline 108 & 180 & -0.090 & 0.000 & -0.024 & -0.002 & 0.001 & -3.12 & -22.30 & 6.69 & 8.94 & 13.23 & 10.36 & 5.76 \\
\hline 108 & 182 & -0.060 & 0.000 & -0.030 & -0.008 & -0.001 & 0.00 & -23.23 & 6.44 & 8.63 & 13.83 & 11.63 & 14.10 \\
\hline 108 & 186 & 0.034 & -0.062 & 0.005 & 0.000 & 0.000 & -5.76 & -23.57 & 7.00 & 7.02 & 14.50 & 8.88 & 0.68 \\
\hline 110 & 154 & 0.319 & 0.000 & -0.052 & -0.012 & 0.006 & -7.43 & -8.77 & 12.31 & 16.15 & 0.62 & -6.48 & -4.27 \\
\hline 110 & 160 & 0.306 & 0.000 & -0.087 & 0.002 & 0.006 & -5.66 & -6.75 & 10.79 & 15.01 & 3.55 & -3.09 & 3.94 \\
\hline 110 & 162 & 0.303 & 0.000 & -0.096 & 0.006 & 0.007 & -0.03 & -6.39 & 10.32 & 14.43 & 4.57 & -1.90 & 9.02 \\
\hline 110 & 164 & 0.293 & 0.000 & -0.101 & 0.014 & 0.003 & -4.93 & -6.71 & 11.41 & 12.32 & 5.74 & -4.55 & 5.87 \\
\hline 110 & 166 & 0.283 & 0.000 & -0.106 & 0.021 & -0.001 & -6.63 & -7.26 & 10.77 & 11.79 & 6.82 & -3.04 & 3.63 \\
\hline 110 & 168 & 0.271 & 0.000 & -0.110 & 0.027 & -0.004 & -6.90 & -8.28 & 10.14 & 11.34 & 7.81 & -1.42 & 1.94 \\
\hline 110 & 178 & -0.145 & 0.000 & -0.032 & 0.008 & 0.006 & 0.00 & -19.31 & 7.24 & 10.50 & 11.24 & 8.63 & 4.61 \\
\hline 110 & 180 & -0.090 & 0.000 & -0.024 & -0.002 & 0.001 & -3.08 & -21.19 & 7.53 & 9.53 & 11.83 & 7.37 & 5.67 \\
\hline 110 & 182 & -0.060 & 0.000 & -0.030 & -0.008 & -0.001 & -0.01 & -22.18 & 7.24 & 9.23 & 12.43 & 8.63 & 13.90 \\
\hline 110 & 184 & 0.000 & -0.002 & 0.000 & 0.000 & 0.000 & 0.00 & -23.43 & 8.03 & 7.84 & 12.86 & 5.36 & 6.25 \\
\hline 110 & 186 & 0.035 & -0.035 & 0.004 & 0.000 & 0.000 & -6.59 & -23.04 & 8.29 & 7.15 & 12.99 & 4.39 & -10.25 \\
\hline 112 & 158 & 0.297 & 0.000 & -0.076 & 0.002 & 0.004 & -8.73 & -9.36 & 12.07 & 16.32 & 0.86 & -5.43 & -2.42 \\
\hline 112 & 160 & 0.295 & 0.000 & -0.088 & 0.006 & 0.005 & -6.01 & -8.62 & 11.50 & 15.94 & 1.79 & -4 & 1.58 \\
\hline 112 & 162 & 0.294 & 0.000 & -0.098 & 0.009 & 0.005 & -0.04 & -7.98 & 11.17 & 15.34 & 2.70 & -3.41 & 6.17 \\
\hline 112 & 164 & 0.283 & 0.000 & -0.103 & 0.017 & 0.001 & -4.70 & -7.77 & 12.10 & 13.50 & 3.88 & -5.50 & 4.78 \\
\hline 112 & 166 & 0.273 & 0.000 & -0.109 & 0.025 & -0.002 & -6.09 & -7.78 & 11.52 & 12.90 & 4.99 & -4.23 & 3.56 \\
\hline 112 & 168 & 0.262 & 0.000 & -0.113 & 0.031 & -0.005 & -6.12 & -8.13 & 10.94 & 12.37 & 6.02 & -2.86 & 2.87 \\
\hline 112 & 186 & 0.052 & -0.007 & 0.005 & 0.001 & 0.001 & -7.35 & -20.94 & 9.80 & 6.86 & 11.35 & 0.18 & -8.67 \\
\hline 112 & 188 & 0.015 & -0.072 & 0.004 & -0.001 & 0.000 & -11.05 & -22.03 & 8.68 & 8.27 & 12.86 & 3.73 & -8.10 \\
\hline 112 & 190 & 0.028 & -0.123 & 0.011 & -0.005 & 0.000 & -10.70 & -21.64 & 9.01 & 6.43 & 13.26 & 2.62 & -14.23 \\
\hline 114 & 160 & 0.284 & 0.000 & -0.089 & 0.010 & 0.003 & -6.36 & -8.58 & 12.08 & 16.84 & 0.28 & & -0.80 \\
\hline 114 & 162 & 0.285 & 0.000 & -0.100 & 0.012 & 0.004 & -0.10 & -7.72 & 11.87 & 16.15 & 1.09 & -4.44 & 3.72 \\
\hline 114 & 164 & 0.272 & 0.000 & -0.105 & 0.020 & 0.000 & -4.62 & -6.79 & 12.53 & 14.68 & 2.27 & -5.85 & 3.62 \\
\hline 114 & 166 & 0.262 & 0.000 & -0.110 & 0.027 & -0.003 & -5.88 & -6.35 & 12.05 & 13.98 & 3.35 & -4.84 & 3.27 \\
\hline 114 & 168 & 0.251 & 0.000 & -0.113 & 0.032 & -0.005 & -6.00 & -6.29 & 11.56 & 13.39 & 4.37 & -3.74 & 3.65 \\
\hline 114 & 170 & 0.231 & 0.000 & -0.106 & 0.031 & -0.006 & -6.70 & -6.67 & 10.97 & 12.96 & 5.37 & -2.33 & 1.60 \\
\hline 114 & 172 & 0.210 & 0.000 & -0.094 & 0.028 & -0.005 & -5.48 & -7.17 & 10.28 & 12.65 & 6.30 & -0.53 & 5.00 \\
\hline 114 & 174 & 0.190 & 0.000 & -0.091 & 0.030 & -0.006 & -1.13 & -8.09 & 9.69 & 12.31 & 7.12 & 1.17 & 6.38 \\
\hline 114 & 176 & -0.153 & 0.000 & -0.022 & 0.005 & 0.002 & -4.32 & -15.07 & 9.74 & 11.44 & 7.68 & 1.02 & 10.60 \\
\hline 114 & 178 & -0.141 & 0.000 & -0.031 & 0.008 & 0.006 & -0.01 & -15.58 & 8.76 & 11.86 & 8.36 & 4.18 & 18.54 \\
\hline 114 & 180 & -0.085 & 0.000 & -0.022 & -0.002 & 0.000 & -3.10 & -17.31 & 9.13 & 10.81 & 9.03 & 2.92 & 9.45 \\
\hline 114 & 182 & -0.059 & 0.000 & -0.029 & -0.007 & -0.001 & -0.01 & -18.41 & 8.78 & 10.49 & 9.65 & 4.11 & 17.10 \\
\hline 114 & 184 & 0.000 & 0.000 & 0.000 & 0.000 & 0.000 & 0.00 & -19.84 & 9.68 & 8.97 & 10.19 & 1.20 & 8.59 \\
\hline 114 & 186 & 0.052 & -0.005 & 0.004 & 0.001 & 0.001 & -7.66 & -18.85 & 10.60 & 7.51 & 10.84 & -1.39 & -6.76 \\
\hline 114 & 188 & 0.032 & -0.064 & 0.006 & 0.000 & 0.000 & -10.96 & -20.07 & 8.81 & 8.65 & 11.22 & 4.00 & -7.58 \\
\hline 114 & 190 & -0.019 & -0.072 & 0.003 & -0.001 & 0.000 & -16.36 & -19.89 & 9.91 & 7.17 & 11.96 & 0.52 & -12.52 \\
\hline 116 & 164 & 0.262 & 0.000 & -0.106 & 0.023 & 0.000 & -4.71 & -3.91 & 13.12 & 15.74 & 0.50 & -6.50 & 1.17 \\
\hline 116 & 166 & 0.252 & 0.000 & -0.112 & 0.030 & -0.004 & -5.81 & -2.26 & 12.76 & 15.04 & 1.56 & -5.78 & 3.84 \\
\hline
\end{tabular}


TABLE I. (Continued.)

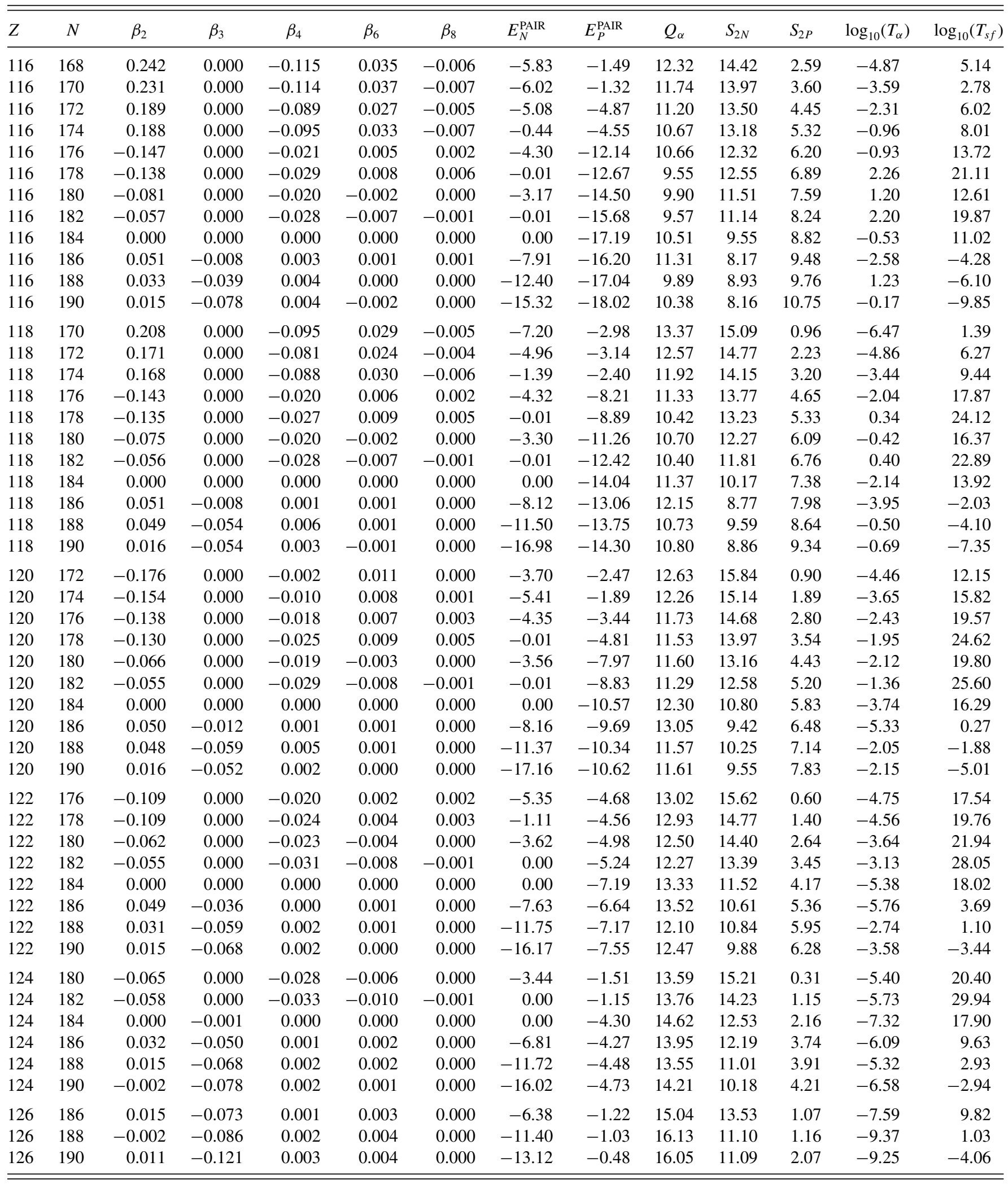

$N=162, \beta_{6}$ becomes almost zero and for $164 \leqslant N \leqslant 174$ we obtain increasing values of $\beta_{6}$ as $N$ increases. For $N \leqslant 176$ we obtain rather small $\beta_{6}$ deformations. Our results for $\beta_{4}$ and $\beta_{6}$ are rather similar to those of Möller et al. [99] and Sobiczewski and co-workers [32]. In contrast to the $\beta_{4}$ and $\beta_{6}$ parameters, $\beta_{8}$ is positive in the region $150 \leqslant N \leqslant 162$. 

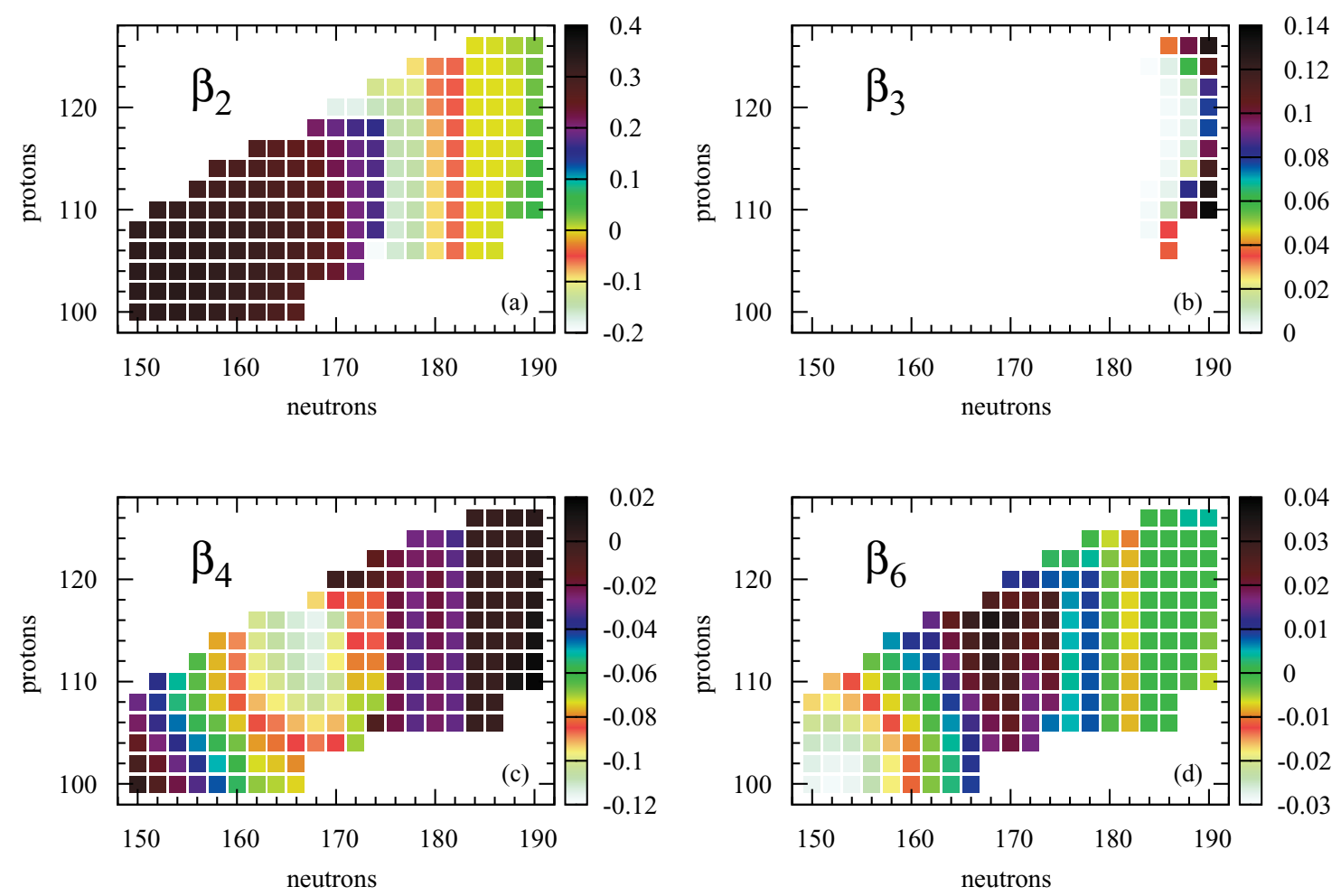

FIG. 3. (Color online) Ground-state deformation parameters: (a) $\beta_{2}$, (b) $\beta_{3}$, (c) $\beta_{4}$, and (d) $\beta_{6}$ for superheavy nuclei. Notice that the deformation scale is panel dependent.

The nuclei with $N=164$ and 166 have either zero or very small $\beta_{8}$ values. The nuclei with $168 \leqslant N \leqslant 174$ have mostly negative $\beta_{8}$ values. With the exception of the $N=178$ isotopes that have positive deformations, all the nuclei with $N \geqslant 176$ have $\beta_{8}$ deformations of almost zero. We have to notice that the maximal absolute values of the deformation parameters decrease with increasing multipolarity, indicating a decreasing relevance. This means that higher multipolarities can be omitted in the minimization of the energy of the ground state in non-self-consistent approximations.

\section{Pairing energies}

In the HFB approach [82], the pairing energy is given by

$$
E^{\mathrm{PAIR}}=-\frac{1}{2} \operatorname{Tr}\left(\Delta \kappa^{*}\right),
$$

with

$$
\Delta_{k_{1} k_{2}}=\frac{1}{2} \sum_{k_{3} k_{4}} \bar{v}_{k_{1} k_{2} k_{3} k_{4}} \kappa_{k_{3} k_{4}}
$$

as the pairing field and

$$
\kappa_{k_{1}, k_{2}}=\left\langle H F B\left|c_{k_{2}} c_{k_{1}}\right| H F B\right\rangle
$$

as the pairing tensor.

The neutron pairing energies of each nucleus are given in the eighth column of Table I and in the upper panel of Fig. 4. The general behavior of these results can be easily understood by looking again at Fig. 2. We know that small pairing energies correspond to situations of low level density of the neutron s.p.e.'s. In particular we expect zero neutron pairing energies at $N=162,178,182$, and 184 . Notice that the fact that for $N=162$ the pairing energies are zero is consistent with the prolate deformation of these nuclei and for $N=178$ with the oblate one. For $N=182$ and 184 we have spherical nuclei. Appropriate neutron shell gaps can be found in Fig. 2 for the mentioned cases. For the same reason large pairing energies are associated with high level density; for example, we find large pairing energies for $N=158,168$, and 190 . We observe that the general pattern, qualitatively, does not depend on the proton number. The proton pairing energies are given in the ninth column of Table I and the lower panel of Fig. 4. In this case the general pattern looks more complicated. For the same proton number some nuclei are prolate, some oblate, and some spherical, and therefore only part of them fit into the proton shell gaps in Fig. 2. Again the small pairing energies found at $Z$ values of 104, 108, 116, 120, and 126 have to do with the low proton level density found in Fig. 2 for those proton numbers. The behavior is different for different isotopes; for example, for $Z=112$, from $N=158$ up to $N=$ 174 we have proton pairing energies of around $-9 \mathrm{MeV}$ and from $N=176$ they are around $-20 \mathrm{MeV}$. The reason for this difference is that in the first interval the nuclei are prolate and in the second they are either oblate or spherical. Similar arguments apply for other cases. It is interesting to notice that with the exception of the few nuclei with $N \geqslant 188$ none of the nuclei studied has absolute values of the neutron pairing energies larger than $9 \mathrm{MeV}$. On the other hand, many of the analyzed nuclei have proton pairing energies much larger than $10 \mathrm{MeV}$. 

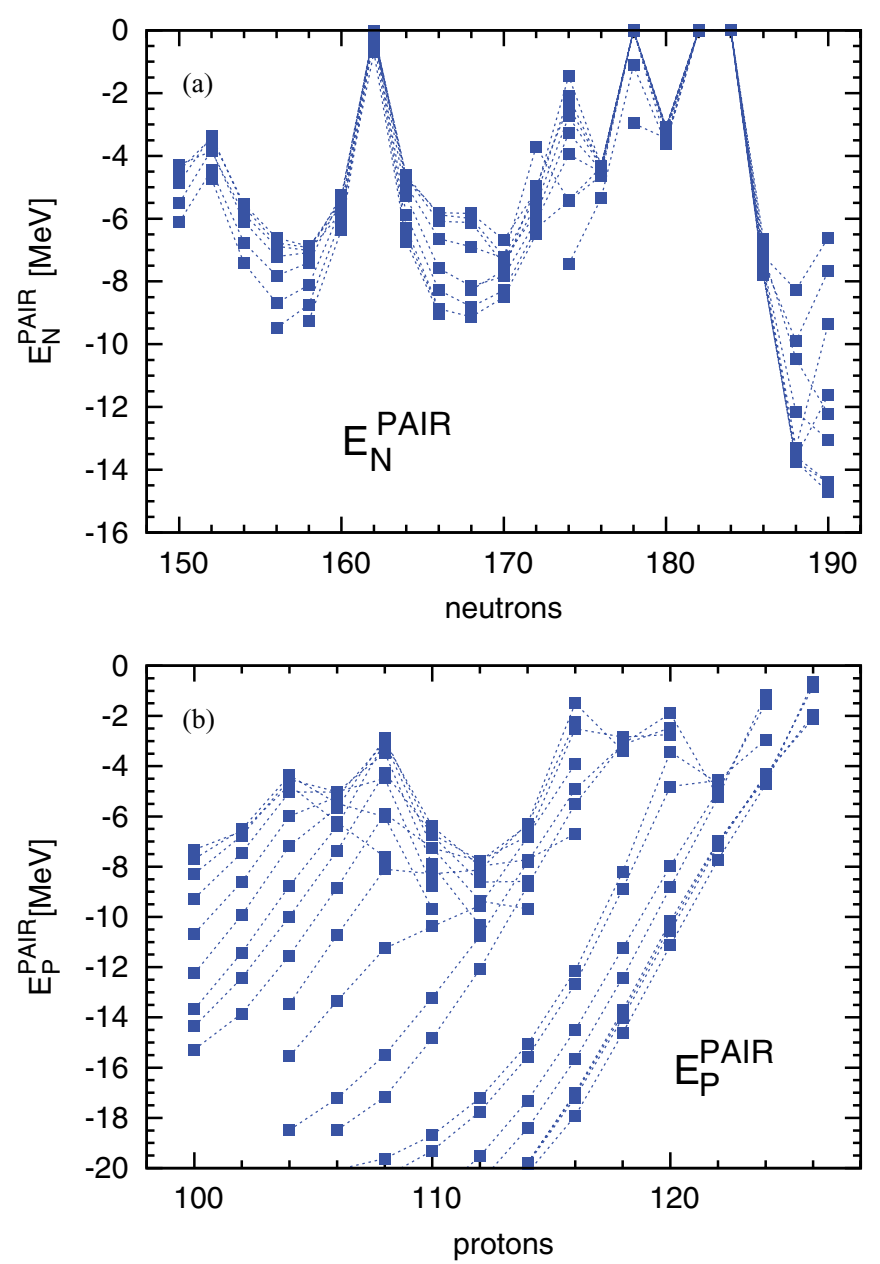

FIG. 4. (Color online) Ground-state pairing energies for superheavy nuclei: (a) protons and (b) neutrons.

\section{D. $Q_{\alpha}$ values}

In Fig. 5 the $Q_{\alpha}$ values are plotted as a function of the neutron number [see Eq. (8)]. The exact numerical values are also given in column 10 of Table I. The patterns displayed by the different isotopes are easily understood by just looking at Eq. (8): minima appear when the mother nucleus is more bound than the average and maxima correspond to more bound daughter nuclei. Thus we observe minima corresponding to the neutron numbers for which energy gaps appear in the singleparticle energies of Fig. 2, namely, 162, 178, and 182-184. For the $N$ values of 164 and 172 there is no structure because the energy gaps that one finds for these neutron numbers are not large enough to provide energy minima at those deformations (see column 3 of Table I). Experimental values [10,94] for some isotopes of the nuclei Fm, No, Rf, Sg, Hs, Ds, 114 116, and 120 are also displayed in the figure. The agreement between theory and experiment is very satisfactory; in most of the cases we obtain a quantitative agreement and for the others at least the tendency is the right one. For the proton dependence we observe a similar situation: we find large energy spacing for the lines whose $Z$ numbers correspond to energy gaps in the single-particle diagram of Fig. 2, for example, Hs, 116, 120 , etc.

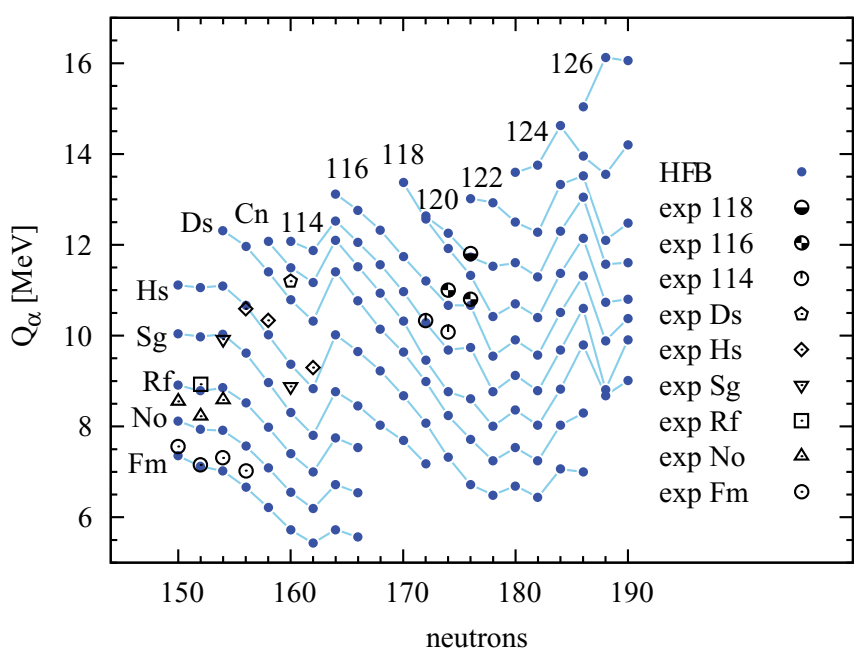

FIG. 5. (Color online) $Q_{\alpha}$ values: theoretical values marked by dots are compared with experimental data [10,94]. The different lines correspond to the indicated isotope.

\section{E. Two-nucleon separation energies}

In Fig. 6 (upper panel), we present the two-neutron separation energy as a function of the neutron number (see also column 11 of Table I). As expected, we obtain a decreasing behavior of $S_{2 N}$ with increasing neutron number since we get closer to the neutron drip line. The smooth decline of $S_{2 N}$ is only disturbed at the neutron numbers corresponding to the single-particle shell gaps 162, 184, etc. The two-proton separation energies are shown, in the lower panel of Fig. 6, as a function of the proton number (see also column 12 of Table I). The general behavior of decreasing $S_{2 P}$ for a given isotonic chain with growing $Z$ illustrates the fact that we are getting closer to the proton drip line. The fact that the $S_{2 P}$ energies present less structure than the $S_{2 N}$ ones is obviously related with the fact that, in the region of interest, the shell gaps in Fig. 2 are smaller for protons than for neutrons. Figure 6 also includes the available experimental values for some $\mathrm{Fm}$ and No isotopes. We observe that in the case of $S_{2 N}$ the theoretical values are around $1 \mathrm{MeV}$ smaller compared to experimental one while in the case of $S_{2 P}$ they are around $1 \mathrm{MeV}$ larger. In both cases, however, the trend is correctly described. This is the well-known binding energy drift that takes place with the D1S parametrization of the Gogny force for most isotopic chains. To correct this drift Chappert et al. [103] have recently devised a new parametrization of the Gogny force, the D1N, which reduces considerably the drift but otherwise keeps the quality of the D1S parametrization. In Table II we have included calculations with the D1N parametrization and we observe a considerable improvement in the agreement. We also include in the table the results of calculations with the D1M parametrization of the Gogny force obtained by including beyond-mean-field effects in the fit [104], which also do not present the mentioned drift.

The D1N and D1M parametrizations also improve slightly the agreement of $Q_{\alpha}$ with the experiment (see Table II), but this effect is much less pronounced than in the case of the 

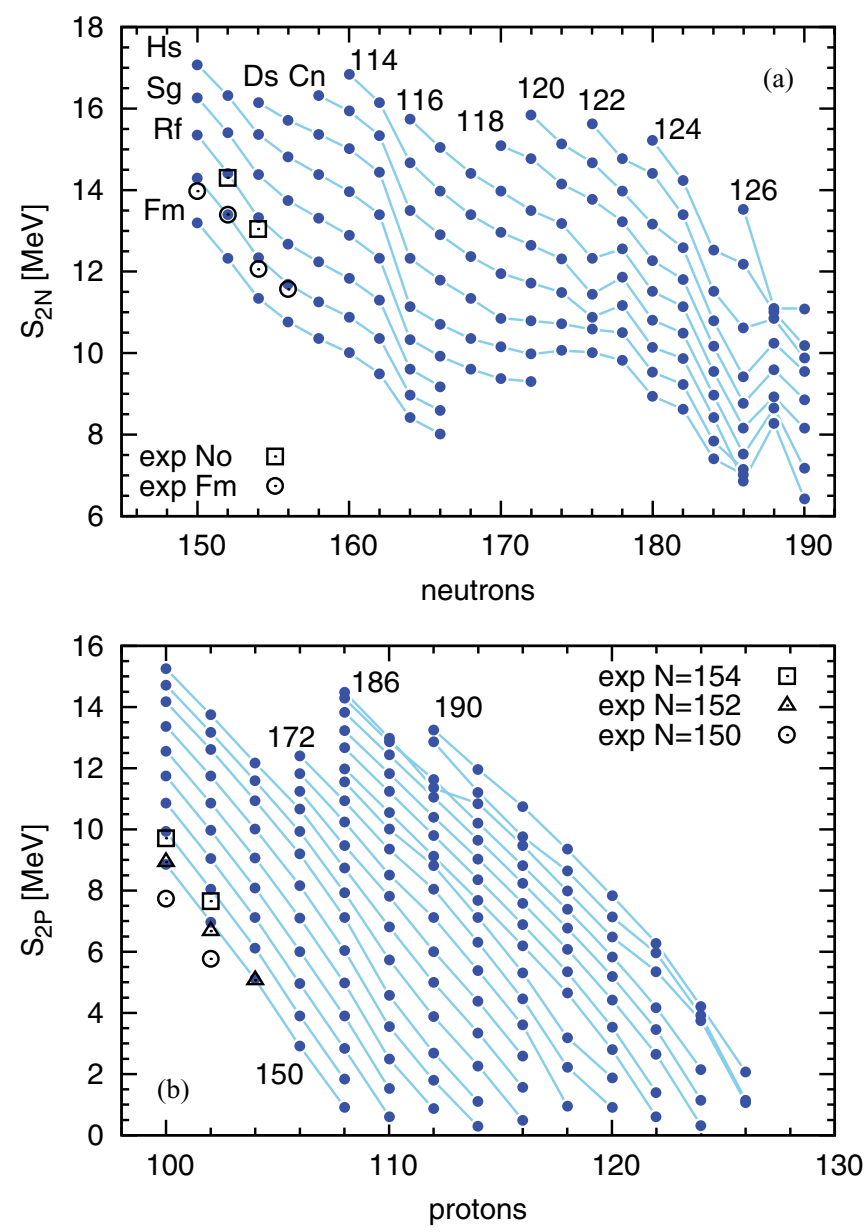

FIG. 6. (Color online) (a) Two-neutron separation energies as a function of the neutron number. (b) Two-proton separation energies as a function of the proton number. The lines represent isotonic chains; the one at the lower left corner corresponds to $N=150$, and the one closer to the upper right corner corresponds to $A=190$. Experimental data are taken from Ref. [94].

separation energies as the influence of protons and neutrons cancel each other.

\section{SPONTANEOUS FISSION}

\section{A. Symmetry-breaking effects in the fission paths}

The large size basis used in these calculations and the large number of nuclei studied prevents us from making a systematic study with totally unrestricted symmetry-breaking wave functions. To clarify the effects of these restrictions we show in Fig. 7, as an example, the behavior of several relevant quantities along the fission path for the nuclei ${ }^{274} \mathrm{Hs}$ (left panel), ${ }^{278} \mathrm{Ds}$ (middle panel), and ${ }^{282} \mathrm{Cn}$ (right panel) in different approximations. Panels (d), in the middle row of Fig. 7, display three different fission paths for each nucleus corresponding to the following constraints on the wave functions: 1 . the axially symmetric $(\gamma=0)$ and reflection-symmetric $\left(Q_{3}=0\right)$ fission path (continuous line), which we shall call in the following the AS-RS path, 2. the axially symmetric and non-reflection-symmetric (AS-NRS) $\left(Q_{3} \neq 0\right)$ path
TABLE II. Two-nucleon separation energies and $Q_{\alpha}$ in Fm isotopes calculated with various Gogny forces compared with experimental (Expt.) data [94] (in units of MeV).

\begin{tabular}{rrrrrr}
\hline \hline & $N$ & D1S & D1N & D1M & Expt. \\
\hline$S_{2 N}$ & 150 & 13.20 & 13.86 & 14.00 & 13.98 \\
& 152 & 12.32 & 13.01 & 13.14 & 13.40 \\
& 154 & 11.35 & 12.13 & 12.23 & 12.06 \\
& 156 & 10.77 & 11.64 & 11.73 & 11.56 \\
$S_{2 P}$ & 150 & 8.86 & 7.80 & 7.62 & 7.74 \\
& 152 & 9.93 & 8.79 & 8.59 & 8.93 \\
& 154 & 10.87 & 9.67 & 9.47 & 9.71 \\
& 156 & 11.74 & 10.50 & 10.31 & 10.43 \\
$Q_{\alpha}$ & 150 & 7.35 & 7.66 & 7.70 & 7.56 \\
& 152 & 7.12 & 7.49 & 7.54 & 7.15 \\
& 154 & 7.02 & 7.38 & 7.48 & 7.31 \\
& 156 & 6.66 & 6.99 & 7.10 & 7.03 \\
\hline \hline
\end{tabular}

(long-dashed lines), and 3. the non-axially-symmetric $(\gamma \neq$ 0 ) and reflection-symmetric (NAS-RS) path (short-dashed lines).

In the ground state these nuclei are well quadrupole deformed, with $Q_{2} \approx 15 \mathrm{~b}$ [see panels (d)], and to fission they have to tunnel through a barrier with a height of several MeV. The barriers represent the potential energy needed to deform the nucleus. They are related, therefore, to the single-particle levels around the Fermi surface available at the corresponding deformation along the path. For the three nuclei the paths in the AS-RS case present two-hump barriers where the height of the second barrier decreases with the mass number while the first one remains more or less constant for ${ }^{274} \mathrm{Hs}$ and ${ }^{278} \mathrm{Ds}$ and increases for ${ }^{282} \mathrm{Cn}$. The width of the barrier in this approach is also similar for the three nuclei. The origin of the two humps can be easily understood by looking at the single-particle energies of Fig. 2. If we follow the neutron Fermi level to the prolate side we find two regions with a clearly developed low level density. The first one at $Q_{2} \approx 15 \mathrm{~b}$ with the shell gaps $N=162$ and $Z=108$ corresponds to the ground-state minimum. On the way from this point to larger deformations we find a larger level density region, which corresponds to the energy increase of the first barrier. Behind that we arrive at the second region at $Q_{2} \approx 32 \mathrm{~b}$, which corresponds to the superdeformed minimum. At even larger deformations one finds a high level density region that provides the energy rise of the second barrier. Finally, above $40 \mathrm{~b}$, we observe intruder states of a high-lying $\nu i_{13 / 2}$ orbital, which could be an indication of the scission point. Incidentally, since the neutron shell gap is at $N=162$ and the proton one is at $Z=112$ the superdeformed minimum is deeper in ${ }^{282} \mathrm{Cn}$ than in the lighter nuclei displayed in Fig. 7.

This behavior changes remarkably in the AS-NRS approach. One can follow in panels (f) the portion of the paths where a lower solution with $Q_{3} \neq 0$ is found. As one can see in panels (f) the octupole degree of freedom plays an important role for quadrupole deformations starting around $Q_{2} \approx 30-35$ b, i.e., close to the superdeformed minima. In fact, in this channel the second hump of the barriers diminishes 

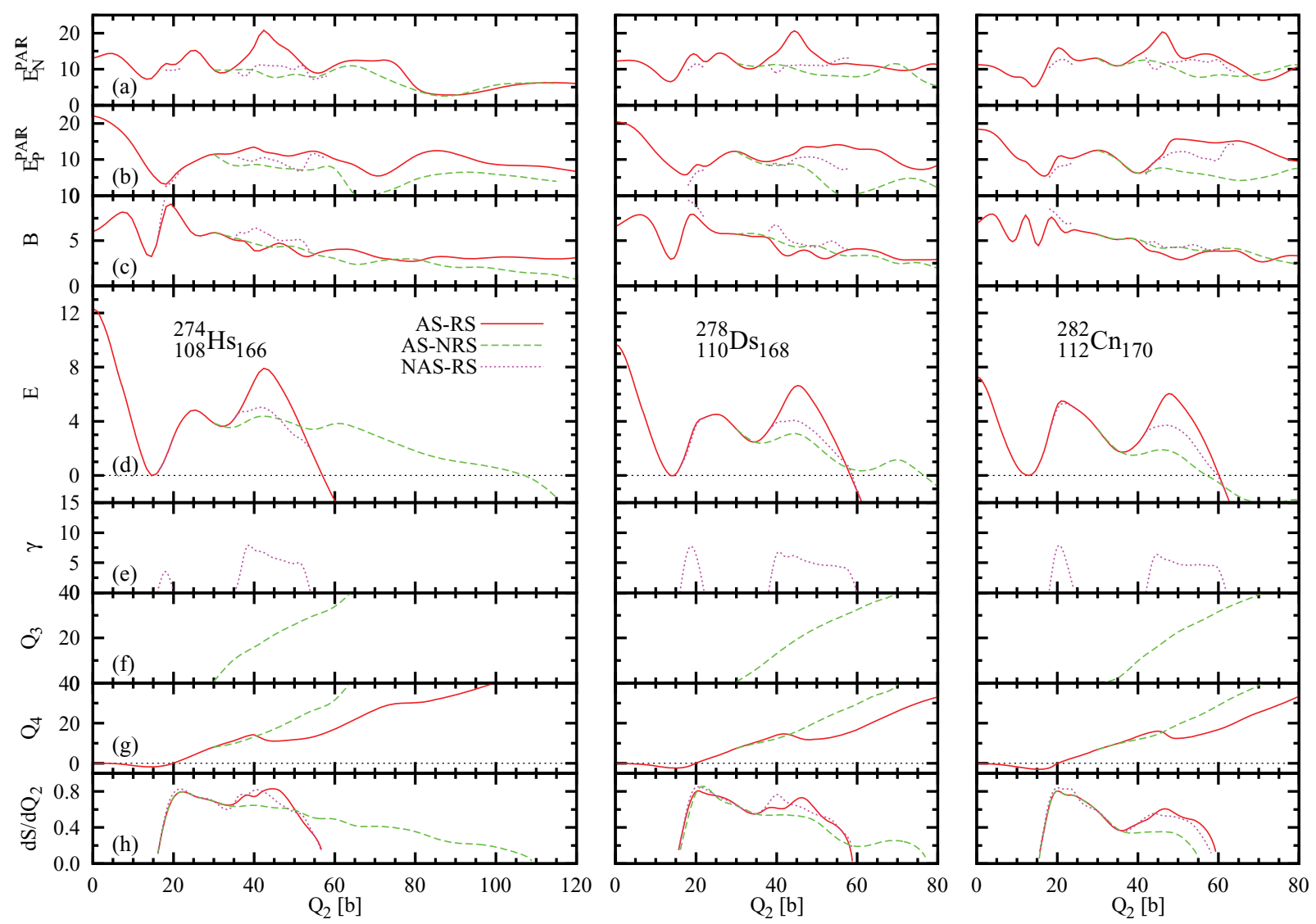

FIG. 7. (Color online) Behavior of several magnitudes for the nuclei ${ }^{274} \mathrm{Hs},{ }^{278} \mathrm{Ds}$, and ${ }^{282} \mathrm{Cn}$ along different fission paths. Neutron and proton pairing energies $E_{P}^{N}$ (a) and $E_{P}^{P}$ (b) are in $\mathrm{MeV}$, (c) inertia parameter $B$ is in $10^{-6} \mathrm{~b}^{-2} \mathrm{MeV}^{-1}$, (d) HFB potential energy is in MeV, (e) triaxial deformation parameter $\gamma$ is in degrees, (f) octupole moment $Q_{3}$ is in $\mathrm{b}^{3 / 2}$, (g) hexadecapole moment $Q_{4}$ is in $\mathrm{b}^{2}$, and (h) the subintegral function of the action integral $d S / d Q_{2}$ is in $\mathrm{b}^{-1}$.

strongly and the paths in this region look like the continuation of the first hump. The AS-NRS path reaches up to large deformations, $Q_{2} \approx 108 \mathrm{~b}$ for ${ }^{274} \mathrm{Hs}$ and $Q_{2} \approx 77 \mathrm{~b}$ for ${ }^{278} \mathrm{Ds}$, and decreases to $Q_{2} \approx 67 \mathrm{~b}$ for ${ }^{282} \mathrm{Cn}$.

We can understand the onset of octupole deformation by looking again at Fig. 2. Here we observe that the $K=1 / 2$ and $K=3 / 2$ levels of the $v k_{17 / 2}$ shell cross the Fermi surface around $26 \mathrm{~b}$, while the $K=5 / 2$ level crosses around $35 \mathrm{~b}$. Interestingly, at these $Q_{2}$ values the $K=5 / 2$ level of the $v h_{11 / 2}$ orbit also approaches the Fermi surface. In Fig. 2 and at zero deformations the $v k_{17 / 2}$ subshell lies at about $0.4 \mathrm{MeV}$ and the $v h_{11 / 2}$ one lies at about $-10.4 \mathrm{MeV}$. The $k_{17 / 2}$ and $h_{11 / 2}$ subshells interact strongly through the octupole interaction, since $\Delta L=\Delta J=3$. That means if we allow for reflection-symmetry breaking we can increase the quadrupole deformation at a lower energy cost [105]. We can observe in Fig. 7 that around these values the AS-NRS fission paths get lower in energy than the AS-RS ones. In the $(\mathrm{g})$ panels we can follow the behavior of the hexadecapole moment along the fission path. In the ground state it is close to zero in both approximations and from this point on it grows linearly with $Q_{2}$ in the AS-NRS approach. In the AS-RS approach, however, first it increases linearly up to the scission point, where a kink is observed followed by a linear increase.
In the NAS-RS path we can observe the effect of the triaxial shapes along the fission paths. In panels (e) we can see the two portions of the trajectory where triaxial solutions are found. The first one, close to the ground state, spans a smaller interval of $Q_{2}$ values than the second one and does not have a large impact on the energy. The second one, as in the AS-NRS case, is relevant around the second hump, causing a significant lowering of its height; i.e., in this case we still have to deal with two humped barriers. The width of the barrier, at variance with the AS-NRS case, is more or less like the AS-RS one.

Though the shape of the barriers is very relevant to calculate lifetimes, one has to consider, however, that other quantities entering in the corresponding formulas, Eqs. (1) and (2), also play an important role. A relevant parameter is the collective quadrupole inertia $B\left(Q_{2}\right)$, since mass parameters are strongly influenced by pairing correlations and these are influenced by the single-particle level density, which we expect to vary along the different fission paths. In panels (a) and (b) of Fig. 7 we display the neutron and proton pairing energies, respectively. We indeed observe big differences in both of them along the portions of the paths where symmetry breaking takes place. In particular, we observe that the AS-RS solutions always provide the largest pairing energies. As we can observe in panels (c), where the $B\left(Q_{2}\right)$ values are plotted 
in the different approaches, in the relevant parts the AS-RS approach provides smaller masses followed by the AS-NRS ones. This implies that not necessarily the smallest fission barriers provide the shortest lifetimes [29]. Since the action $S$ in Eq. (2) can be seen as a line integral of the function $d S\left(Q_{2}\right) / d Q_{2}=\left\{2 B\left(Q_{2}\right)\left[V\left(Q_{2}\right)-E_{0}\right]\right\}^{1 / 2}$, the area of the surface delimited by this line and the $x$ axis provides the value of the action $S$. In panel (h) we display $d S\left(Q_{2}\right) / d Q_{2}$ in the three approaches. For the nucleus ${ }^{274} \mathrm{Hs}$ we find that though the NAS-RS fission path has a smaller fission barrier than the AS-RS one, the actions $S$ for both approaches are very close. The actual values of $S$ are 26.69 (AS-RS) and 26.49 (NAS-RS), while in the AS-NRS case we obtain a much larger value, namely 41.66 . In the nucleus ${ }^{278} \mathrm{Ds}$, the fission paths alone would predict that the AS-NRS and the NAS-RS approaches would provide much shorter lifetimes than the AS-RS one. However, in panel (h) one finds that the three areas look rather similar. Actually, the precise numbers 25.88 (AS-RS), 26.97 (AS-NRS), and 26.49 (NAS-RS) show this to be the case. Lastly, for ${ }^{282} \mathrm{Cn}$, the prediction of the fission paths is more or less in accordance with the one of panel (h) and the actual numbers 23.34 (AS-RS), 18.32 (AS-NRS), and 22.52 (NAS-RS) corroborate that. We can conclude that the restriction to axially symmetric paths is, in general, a good approximation, though as we we will see later one can find some exceptions.

The low and short non-reflection-symmetric barrier in nuclei with $N \geqslant 170$ makes the most probable fission through octupole-deformed shapes. In these nuclei we expect to find an asymmetric mass distribution of fission fragments [106].

In the one-dimensional fission paths plotted in Fig. 7 we find crossings between the two paths, giving the impression that one could switch from one path to the other without further problem. However, if we look at a higher dimensional plot one can see that this is not the case. To illustrate this point we have drawn in Fig. 8 potential energy contour lines versus the quadrupole moment, $Q_{2}$, and the octupole moment, $Q_{3}$, for the nuclei ${ }^{274} \mathrm{Hs}$ and ${ }^{282} \mathrm{Cn}$. In this figure we can follow the AS-RS and the AS-NRS paths of Fig. 7 for the respective nucleus. The AS-RS path corresponds to $Q_{3}=0$ and goes along the $x$ axis and the AS-NRS one goes along the bullets. It is interesting to see how the self-consistent path goes along a valley in both nuclei. We can also see that no alternative paths are present. In the ${ }^{274} \mathrm{Hs}$ case we find that, at $Q_{2}=50 \mathrm{~b}$ where both paths seem to cross in Fig. 7(d), in reality both paths are separated by a 4 - to $5-\mathrm{MeV}$-high barrier.

\section{B. Fission barriers in the axially symmetric approaches}

In the following to perform a systematic description of the fission barriers of the 160 SHEs, we restrict ourselves to the axial approximation in which we have performed two kinds of calculations, namely, the reflection-symmetric, AS-RS, and the non-reflection-symmetric, AS-NRS. All the fission barriers are presented in Figs. 9-13.

In Fig. 9 we present the fission barriers for the isotopes of the elements Fm, No, and Rf for quadrupole values from -20 to $80 \mathrm{~b}$ (continuous line for AS-RS and dashed line for AS-NRS). We first discuss the AS-RS results. In panel (a) we present the
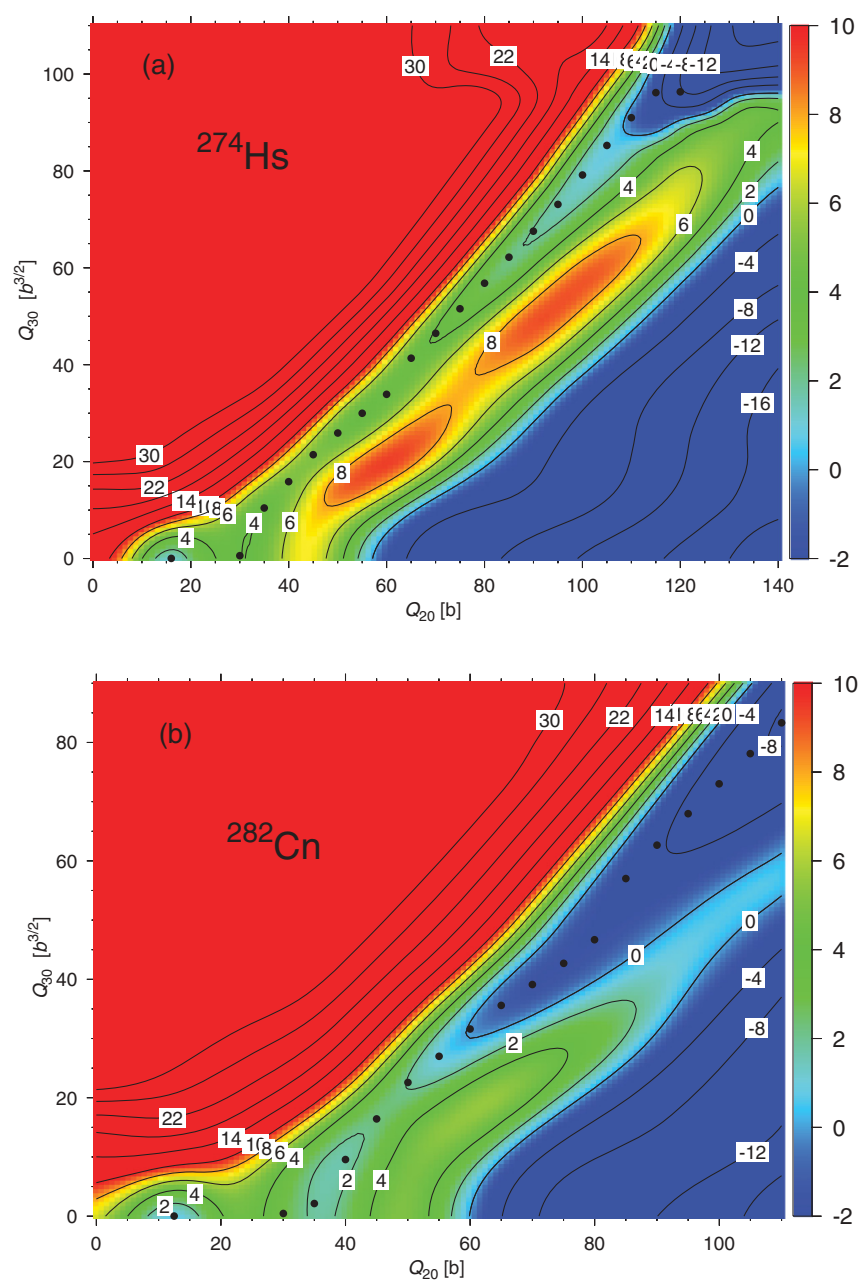

FIG. 8. (Color online) The PES as a function of quadrupole and octupole moments for (a) ${ }^{274} \mathrm{Hs}$ and (b) ${ }^{282} \mathrm{Cn}$. The energy origin has been set at the energy minimum.

Fm results for neutron number 150 up to 166. All isotopes present a well-prolate-deformed minimum around $15 \mathrm{~b}$. In addition in the lighter isotopes a shallow superdeformed (SD) minimum appears around $50 \mathrm{~b}$, at $N=156$ we find a very flat minimum, and for the heavier isotopes no SD minimum is found. The common characteristic of these nuclei is the presence of a big broad barrier. For $N=150$ the barrier is centered at $Q_{2}=30 \mathrm{~b}$, has a height of about $12 \mathrm{MeV}$, and has a width of $18 \mathrm{~b}$. With increasing neutron number the center of the barrier shifts to larger deformations and the height diminishes. For $N=166$ the center is around $Q_{2}=38 \mathrm{~b}$ and the height is about $8 \mathrm{MeV}$. In the heavier isotopes we find some structure in the first barrier, namely, the development of a shoulder around $Q_{2}=27 \mathrm{~b}$ with increasing neutron number. The presence of a SD minimum in the lighter isotopes drives the existence of a second barrier. Since the minima are rather shallow the second barriers are broad but not high. These properties will contribute in general to a tendency of shorter lifetimes with increasing neutron number, though the particular behavior must be analyzed case by case.

The fission paths for the No isotopes are shown in panel (b) for the same neutron numbers as the Fm case. The structures of 


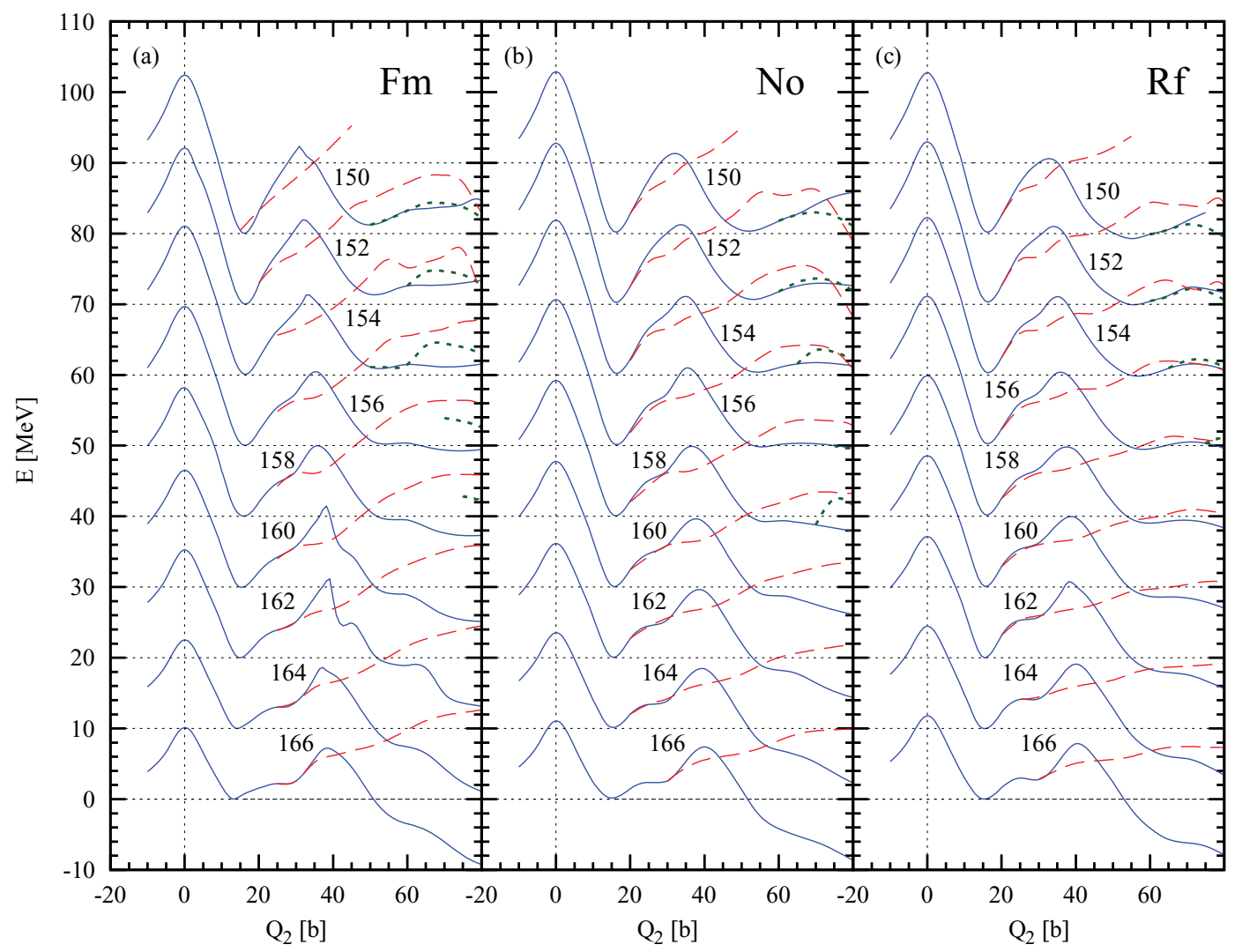

FIG. 9. (Color online) Fission barriers for the nuclei Fm, No, and Rf along the AS-RS (continuous lines) and the AS-NRS path (long-dashed lines). The short-dashed lines in the lighter isotopes for $Q_{2}>50 \mathrm{~b}$ correspond to the elongated fission paths. For clarity reasons in the ordinate axis the ground-state energy of each isotope has been set to zero and shifted by $10 \mathrm{MeV}$ in each isotope.

the first barriers look roughly like the ones in Fm. An important point is the fact that the SD minimum is somewhat lower in energy than in the Fm case and its role is therefore much less relevant. The results for the $\mathrm{Rf}$ isotopes are displayed in panel (c). The tendency observed in the No isotopes is reinforced, the SD minimum gets even deeper, and the second barrier disappears for most isotopes. This trend will provide in general shorter lifetimes for Rf than for No and for No shorter than for Fm. These are general tendencies but since the lifetimes are very sensitive to small energy differences along the fission path, to make quantitative predictions calculations involving also collective inertia have to be performed.

Let us now describe the AS-NRS results. For all isotopes of the three elements, the fission barriers are much larger along the non-reflection-symmetric paths than along the reflectionsymmetric path. The lifetimes are therefore considerably longer in the AS-NRS path than in the AS-RS one. Fission in the AS-NRS mode is completely impossible.

An exhaustive discussion of the fission barriers of the nuclei ${ }^{254} \mathrm{Fm},{ }^{256} \mathrm{Fm},{ }^{258} \mathrm{Fm},{ }^{258} \mathrm{No}$, and ${ }^{260} \mathrm{Rf}$ can be found in Ref. [57]. In these nuclei the "elongated fission" mode can be observed. This mode is connected to octupole-deformed fission paths which start at $Q_{2}>50 \mathrm{~b}$ (see the short-dashed lines in Fig. 9). We have not observed the "elongated fission" in heavier nuclei and therefore we will not discuss it here.

In Fig. 10 we present the paths for the nuclei of Sg, Hs, and Ds elements. In panel (a) we display the results from
$N=150$ up to $N=174$ for the $\mathrm{Sg}$ isotopes. These nuclei have a trend somewhat different from the preceding ones: the SD minimum does not play a relevant role in the fission process since it is always deeper than the ground state and, in fact, if the SD minimum were not that shallow it would be the ground state for the lightest $\mathrm{Sg}$ isotopes. Furthermore, the barriers get flatter, most of them being lower than $10 \mathrm{MeV}$. Apart from this feature the situation for the isotopes $N=150-166$ is similar to the nuclei Fm, No, and Rf; i.e., the ground states are prolate deformed $\left(Q_{2}=15 \mathrm{~b}\right)$ with large negative deformation energies and high barriers at larger $Q_{2}$ values. For $N=$ 168-174 the situation changes very fast, and the following properties get reinforced as the neutron number increases: the ground states get less deformed, the shoulders around $Q_{2}=30$ $\mathrm{b}$ get deeper and become real minima, and as a consequence the original barrier becomes a two-humped one. The height of the spherical maximum decreases and a coexisting oblate minimum develops at $Q_{2}=-10 \mathrm{~b}$. In particular for $N=172$ the oblate and prolate minima are degenerated. We therefore expect a strong reduction in the fission lifetime of heavier isotopes as compared to the lighter ones.

In panel (b) the $N=150$ up to $N=186 \mathrm{Hs}$ isotopes are shown. In the lighter isotopes the fission barriers are flatter and the SD minima are deeper than in the corresponding $\mathrm{Sg}$ isotones. The effect of the SD minima on the fission process is much smaller. Furthermore, the second fission barrier develops much earlier. Since the single-particle energies of Fig. 2 are for 


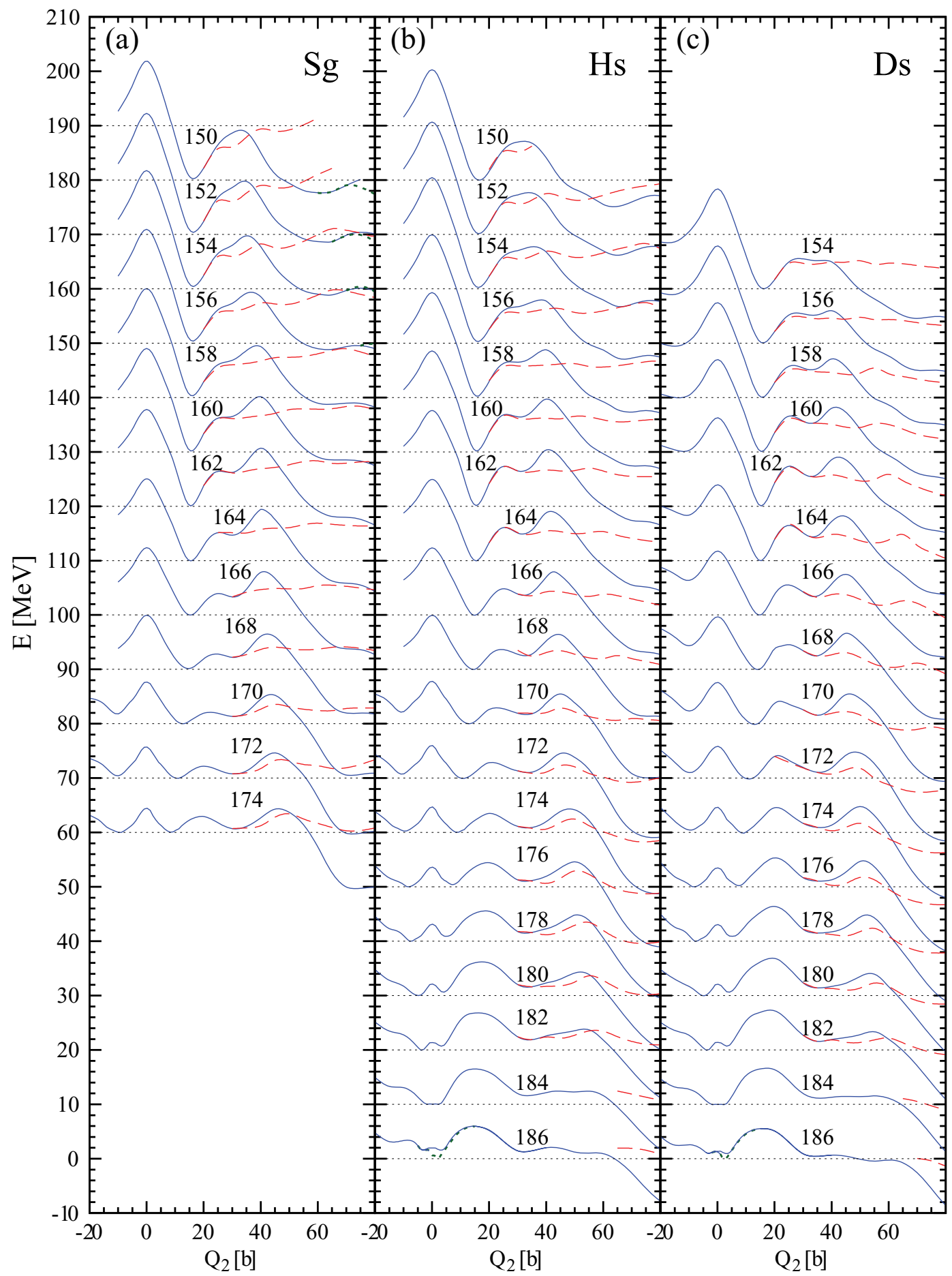

FIG. 10. (Color online) Fission barriers for the Sg, Hs, and Ds isotopes along the AS-RS (continuous lines) and the AS-NRS path (dashed lines). The green short-dashed lines around the ground state for $A>184$ correspond to NRS solutions. For clarity reasons the ground-state energy of each isotope has been set to zero and shifted by $10 \mathrm{MeV}$ in each isotope in the ordinate axis.

${ }^{270} \mathrm{Hs}$ we can refer to the general discussion of Sec. IV A for the shape of the fission path in this nucleus. Aside from these facts the $\mathrm{Hs}$ isotopes from $N=150$ up to $N=174$ behave to a large extent like the corresponding isotones of the $\mathrm{Sg}$ isotopes. For $N=176$ the oblate minimum becomes the ground state and the two humps of the fission barrier are very similar in size and height (about $5 \mathrm{MeV}$ ). With increasing number of neutrons, the prolate minimum shifts toward $Q_{2}=0$ and the spherical minimum becomes the ground state at the $N=184$ shell closure. As a consequence, the two humps separate from each other, the outer one shifting to larger $Q_{2}$ values and the inner one to smaller ones. At the same time the inner barrier 


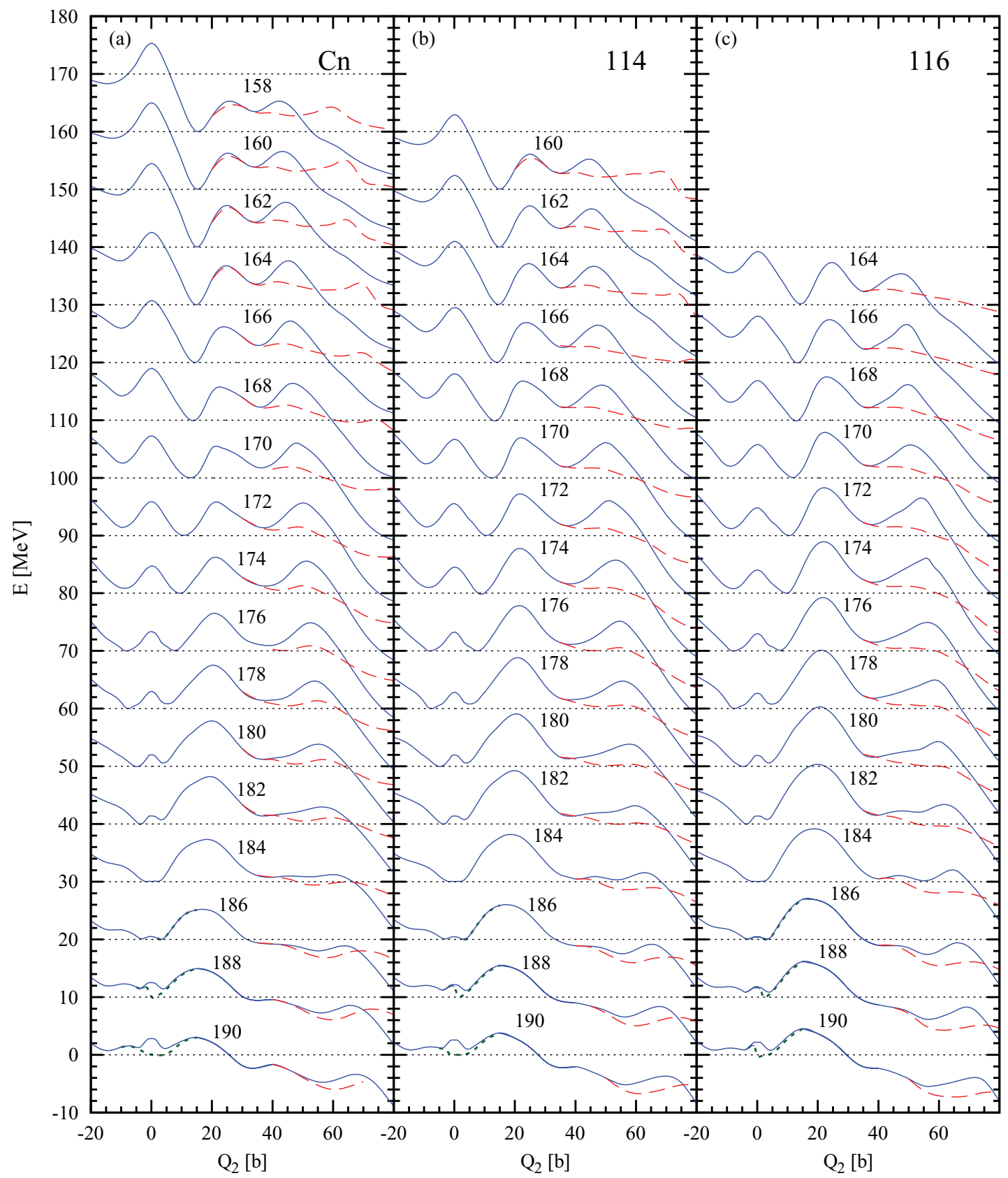

FIG. 11. (Color online) Same as Fig. 10, but for the $\mathrm{Cn}$ and $Z=114$ and 116 isotopes.

gets bigger and the outer smaller. From this behavior one expects the lifetimes for fission to be smaller around neutron number 170-174.

The nucleus ${ }^{294} \mathrm{Hs}$ is octupole deformed in its ground state (see Fig. 3). In the self-consistent $Q_{2}$-constrained calculations this nucleus remains weakly octupole deformed up to $Q_{2}=$ $10 \mathrm{~b}$, where it turns reflection symmetric (see short dashed part of the fission path). Since the fission fragments at the scission point are characteristic of reflection-symmetric fission we still denote this mode as RS fission. The same situation is found with the $N>184$ isotones for the heavier SHEs. The paths along $Q_{3} \neq 0$ close to the ground state are plotted with short dash lines in the corresponding figures.
The Ds results for the isotopes $N=154$ up to $N=188$ are displayed in panel (c). The main characteristics of these fission paths are the following: For the lighter isotopes the fission barriers are flatter than for the corresponding isotones in $\mathrm{Hs}$ and $\mathrm{Sg}$. For the light and medium-mass isotopes, for a given isotone number, we find an increase of the first barrier moving from $\mathrm{Sg}$ to $\mathrm{Hs}$ and from this to Ds. The opposite effect is observed for the second barrier; in particular, for the very heavy isotopes this barrier disappears at the highest neutron number studied.

For the AS-NRS results of Fig. 10 and for the Sg isotopes, we find that though the AS-NRS fission barriers are smaller than the AS-RS ones for medium $Q_{2}$ values, for larger ones 


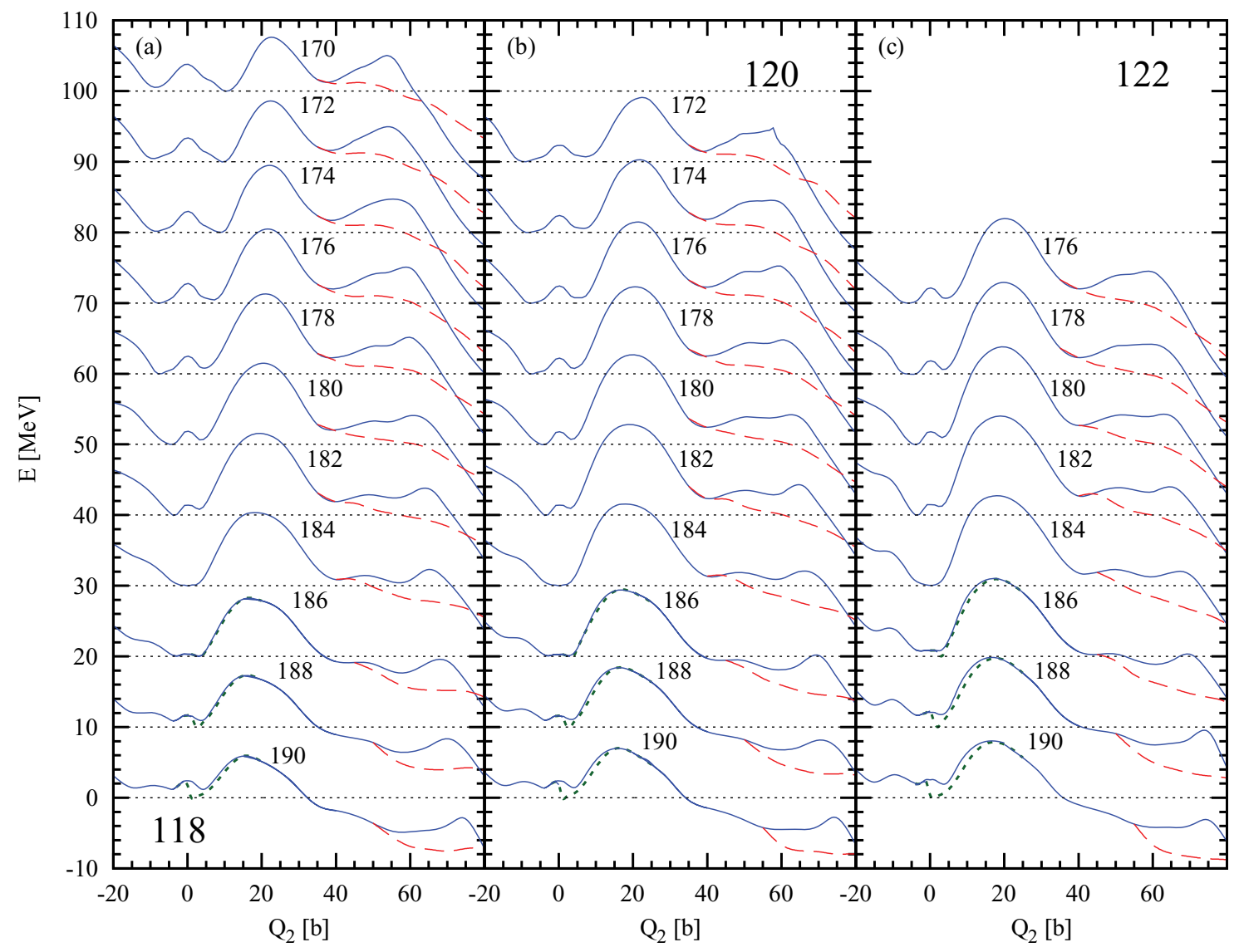

FIG. 12. (Color online) Same as Fig. 10, but for the $Z=118,120$, and 122 isotopes.

they are much higher and as a result the non-reflectionsymmetric fission paths are not favored compared with the reflection-symmetric ones. For the $\mathrm{Hs}$ isotopes the same behavior as the $\mathrm{Sg}$ isotopes is observed: for lighter isotopes up-bending tails of the fission paths make the AS-NRS paths very unfavored, but for medium-heavy $N$ values the tails bend downward and though the path is longer the second barrier is smaller for the AS-NRS path than for the AS-RS one, i.e., around $N=170$ and above the AS-NRS path may compete with the AS-RS one (cf. Fig. 8). For the heaviest Hs isotopes in the AS-RS approach the second barrier decreases considerably and the AS-NRS barrier becomes longer than the other one. For the Ds isotopes the AS-NRS path is even more favorable because the down-bending tendency gets reinforced and we have some AS-NRS paths which are clearly favored, for example for the nuclei with $N=170-176$. For larger $N$ values the vanishing of the second barrier in the AS-RS case again favors this approach. Notice that in general octupole effects set in for larger $Q_{2}$ values as the neutron number increases.

In Fig. 11 we present the fission paths for the isotopes of the elements $\mathrm{Cn}$ and $Z=114$ and 116. As before, we first discuss the AS-RS paths. In panel (a), where the isotopes $N=160-188$ of $\mathrm{Cn}$ are shown, we observe that compared with the corresponding isotones discussed before, the second hump of the barriers is lower and that the slopes of the tails of the fission paths are more pronounced. These facts point to shorter lifetimes of the $\mathrm{Cn}$ isotopes compared with lighter isotones. In panel (b) of the figure the $Z=114$ isotopes are shown. The main difference with respect to the $\mathrm{Cn}$ isotopes is the increase of the first hump and the decrease of the second one. In the isotopes with one-hump barriers these are higher and a bit broader than for the corresponding isotones in $\mathrm{Cn}$. Altogether, it seems that, in general, the lifetimes of the $Z=$ 114 isotopes will be somewhat longer than the one at the corresponding $Z=112$ isotones. In panel (c) we display the $Z=116$ isotopes. Here the same trend as in the previous nuclei is observed: a reinforcement of the tendency to increase the first hump of the double-humped barriers and in the case of only one hump an increase of this.

For the AS-NRS results for $\mathrm{Cn}$ and the $Z=114$ and 116 elements, we find that the onset of octupolarity is energetically favored after the level crossing of the "higher shells" and with the exception of the lightest isotopes the barriers are much smaller in the AS-NRS path. This is due to the disappearance of the second hump of the barrier; i.e., in the AS-NRS path we have only one-humped barriers. We expect therefore a shortening of the fission lifetimes along these paths.

In Fig. 12 the results for the $Z=118,120$, and 122 isotopes is shown. The same tendency as before is observed in the AS-RS calculations: larger first barriers as $Z$ increases. The role of the octupole degree of freedom is also relevant and all AS-NRS fission paths have smaller fission barriers. The same comments also apply to the $Z=124$ and 126 isotopes in Fig. 13. 


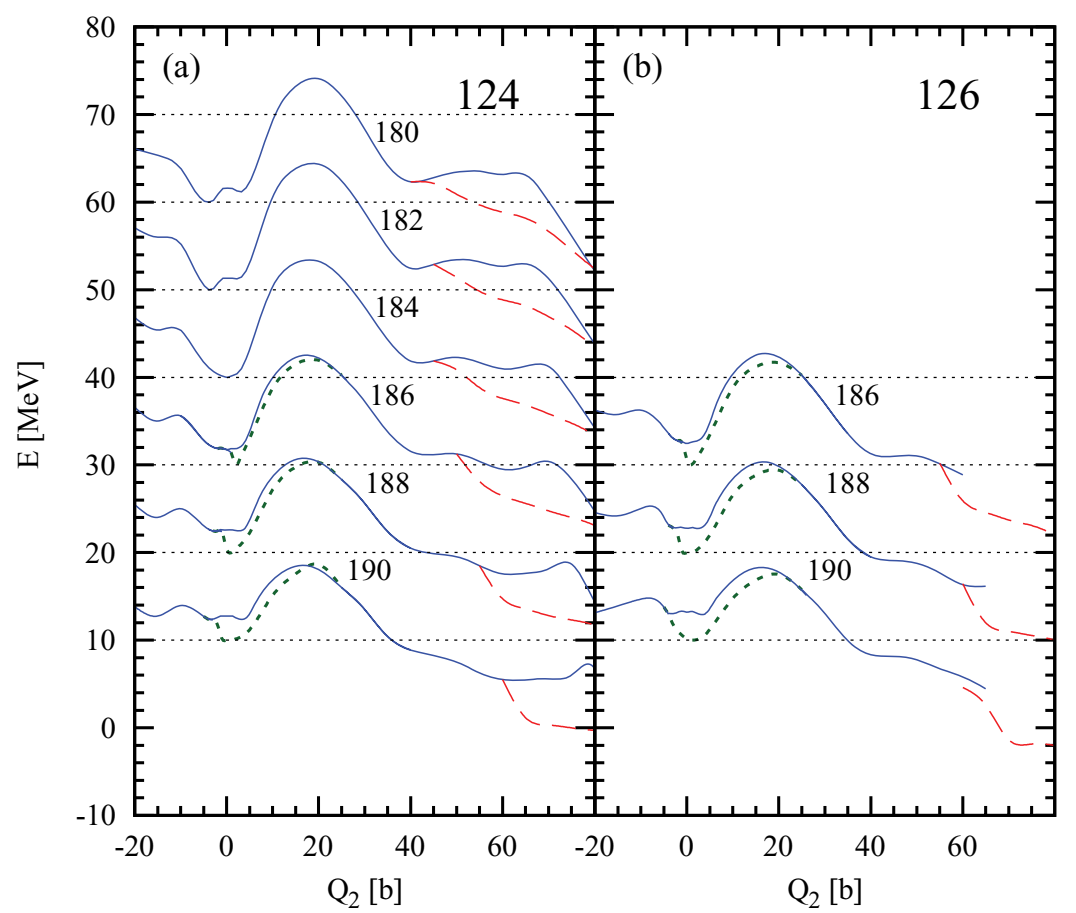

FIG. 13. (Color online) Same as Fig. 10, but for the $Z=124$ and 126 isotopes.

\section{HALF-LIVES OF SHES}

One of the key issues in the theoretical description is the prediction of the decay modes and the half-lives of the SHEs. The agreement between the experimental data and the theoretical predictions not only affirms their quality but may also be a criterion for the identification of a particular isotope synthesized in the corresponding fusion reaction. Moreover, since the contemporary experimental techniques do not allow the detection of nuclei with half-lives shorter than $T=10 \mu$ s one must estimate which isotopes can survive long enough to be detected and the way in which they disintegrate. Therefore, the half-lives of the two main competing processes, spontaneous fission and $\alpha$ emission, should be evaluated. The shortest half-life determines the dominant decay channel and the total half-life. If the branching ratio between two modes is equal to $50 \%$ the logarithm of the total half-life would be smaller than the shorter partial half-life by not more than 0.3 . Differences in half-lives between two modes of one order of magnitude lead to a logarithm of the total half-life only 0.05 smaller than the logarithm of the half-life of the fastest decay.

The half-lives for $\alpha$ decay and spontaneous fission calculated in the HFB theory are collected in the last two columns of Table I. For an easier analysis these data are also presented in Fig. 14 where the isotopic chain of each element is shown in a separate panel. In some nuclei two fission half-lives can be calculated along paths leading to distinct fragment mass asymmetry. Both solutions are depicted in Fig. 14 and discussed below; however, in Table I only the shorter half-life of the dominant mode is given. The available experimental data are also plotted in Fig. 14.

A first look at the panels of Fig. 14 reveals similar tendencies for the different isotopic chains. It is easy to distinguish the intervals of neutron number where common features are characteristic for many elements despite differences in the absolute values. Therefore we will discuss our results collected in groups of similar neutron number, starting from the lighter ones.

As we have seen in the discussion of the last section NRS effects influence the fission paths, and thereby the fission halflives, in three $Q_{2}$ regions: 1 . For small $Q_{2}$ values, $Q_{2}<20$ b, they affect nuclei with octupole-deformed ground states, i.e., nuclei with $N>184$. 2. Starting at medium $\left(Q_{2}>20 \mathrm{~b}\right)$ and extending up to large $Q_{2}$ values, these effects are present in all nuclei. 3. For larger $Q_{2}$ values, $Q_{2}>50 \mathrm{~b}$, they appear in the light isotopes of the elements $\mathrm{Fm}$, No, Rf, and $\mathrm{Sg}$. According to these effects we are using three symbols in Fig. 14 for the fission half-lives depending on the paths used in the calculations: AS-RS (upward-pointing triangles) includes the genuine AS-RS ones plus those with octupole effects close to the ground state. The reason for including the latter under an "AS-RS" denomination is that at $Q_{2} \approx 20$ b the nucleus takes an AS-RS shape and the fission takes place exactly in the same way as in the pure AS-RS case with symmetric fragment mass distribution. AS-NRS (downward-pointing triangles) denotes the "real" non-reflection-symmetric paths leading to fission with fragments of different masses, which correspond to the long dashed lines in the fission barrier plots. AS-RS/NRS (stars) labels the paths where the first part is AS-RS and only for $Q_{2}>50 \mathrm{~b}$ one follows the non-reflection-symmetric branch.

\section{A. The region $150 \leqslant N \leqslant 162$}

The first characteristic region covers the lighter isotopes with $150 \leqslant N \leqslant 162$, clearly delimited by the $N=162$ deformed "shell closure." In this range of $N$ the half-lives for $\alpha$ emission increase monotonically with the neutron number in 


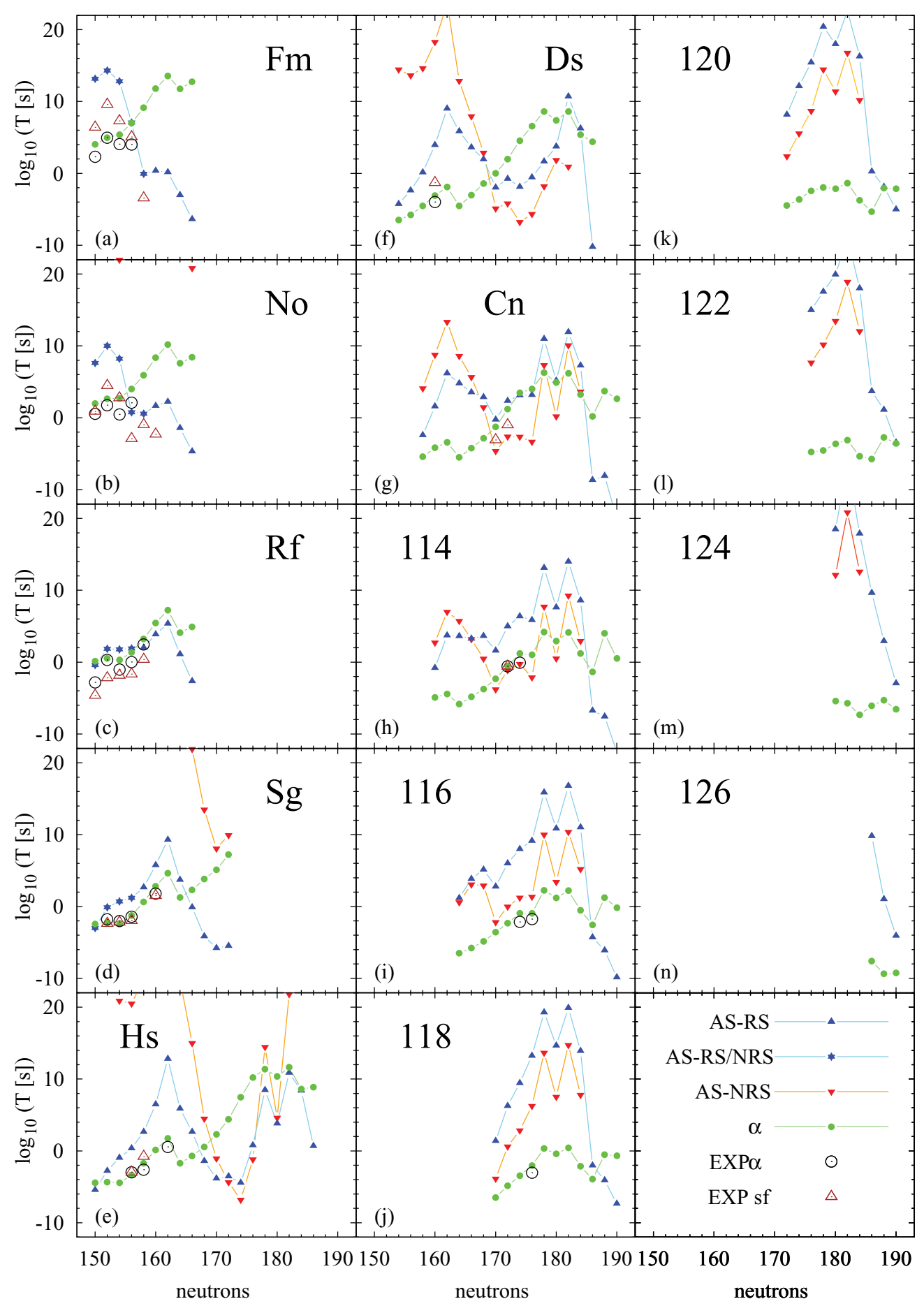

FIG. 14. (Color online) The spontaneous fission and the $\alpha$-emission half-lives in SHEs. Experimental data are taken from Refs. [9,10,107].

all elements from Fm to $Z=114$. It can be also noticed that an increase of the proton number by two units leads to the decrease of the $\alpha$ half-lives by two or three orders of magnitude. As we will also see for the other regions, this tendency is a direct consequence of the calculated values of $Q_{\alpha}$ shown in Fig. 5 and is consistent with well-known properties of $\alpha$ decay in heavy nuclei.

The three lightest Fm and No isotopes comprise a specific group of nuclei in which the HFB calculations predict relatively long fission half-lives. The small fission probability is the consequence of the shape of the fission barrier in these nuclei, which extends to large quadrupole deformation. In the AS-RS path the second barrier extends up to $120 \mathrm{~b}$, providing very long half-lives. A somewhat shorter half-life is obtained in the AS-NRS approach where the barrier is constructed from two humps: a reflection-symmetric one and a non-reflectionsymmetric one starting at $Q_{2} \approx 60 \mathrm{~b}$ (Fig. 9). In the slightly heavier isotopes the second non-reflection-symmetric barrier 
also can be noticed in the PES (Fig. 10), but in contrast to the group of lighter Fm and No nuclei the second, superdeformed minimum has an energy below the ground state. Thus the second hump does not affect the barrier tunneling but it governs the fragment mass asymmetry. In these nuclei the reduction of the width of the barrier leads to a decrease of the half-lives by a few orders of magnitude, which can be seen in the Fm and No isotopes with $N \geqslant 156$ as well as the $\mathrm{Rf}$ and the $\mathrm{Sg}$ isotopes. All nuclei with the second non-reflection-symmetric hump leading to "elongated fission" [57] (i.e., Fm and No with $N \leqslant 158$ as well as $\mathrm{Rf}$ and $\mathrm{Sg}$ with $N \leqslant 156$ ) are marked in Fig. 14 by blue stars.

In the heavier isotopes of the $150 \leqslant N \leqslant 162$ interval, where only the single reflection-symmetric barrier remains, one observes a rise of the fission half-lives with the neutron number. The slope of this trend changes from an almost flat dependence in Fm to a very steep one in $\mathrm{Sg}$, Ds, Cn, and $Z=114$. This trend is caused by a broadening of the barrier, which eventually becomes a two-humped one (Figs. 9, 10, and 11).

The local maximum found in all elements in the partial half-lives at $N=162$ with respect to $\alpha$ and fission decays indicates the special character of this neutron number in the chart of nuclides (see also Fig. 2) These isotones are the most stable in the close vicinity and hence, in many papers $N=162$ is called a "deformed magic number." This name stresses the significant difference from the classical magic nuclei, which are spherical in their ground states.

Many experimental data coming from the "cold fusion" reactions are available in the region of isotopes with $N \leqslant 162$ $[9,107]$. The agreement of the $\alpha$-decay half-lives with the theoretical predictions is noticeable, although some discrepancies are observed. Most calculated fission half-lives overestimate the experimental data. The agreement would be better if triaxial effects were taken into account in the saddle point of the first barrier. The consideration of the $\gamma$ deformation in the calculations of nuclei in the Fm region reduces the barrier heights by around $2 \mathrm{MeV}$, decreasing thereby the theoretical fission half-lives by around two orders of magnitude [57]. At variance with the discussion of Sec. IV A in these nuclei this reduction is not fully compensated by an increase of the inertia parameters. Therefore discrepancies between the theory and the experiment diminish considerably. It should be pointed out that the fastest decay is properly predicted in all cases. Furthermore, when the experimental data for $\alpha$ emission and fission provide comparable decay probability for both processes the theoretical predictions are also similar for both partial half-lives. Almost all nuclei in this region do have half-lives long enough to be considered of experimental interest.

\section{B. The region $164 \leqslant N \leqslant 178$}

The next region covers nuclei with neutron number from $N=164$ to $N=178$. At $N=164$ a kink of two to four orders of magnitude in the $\alpha$-decay half-lives can be observed corresponding to a local maximum in $Q_{\alpha}$ (see Fig. 5). The increase of the neutron number for a given isotope causes a linear growth in the $\alpha$ half-life following the tendency already observed in the lighter nuclei. The half-life rises up to the neutron number $N=178$ where the locally longest living isotopes are found (see also Fig. 5). They reach values from $T=0.1 \mathrm{~s}$ in $Z=118$ to $T=10^{11} \mathrm{~s}$ in Hs. Again, the half-lives calculated for heavier isotones are smaller and, consequently, $\alpha$ emission becomes the most probable decay channel in the proton-rich nuclei.

The AS-RS fission half-lives for nuclei with $164 \leqslant N \leqslant$ 178 behave completely different from those for $150 \leqslant N \leqslant$ 162. After passing the maximum at $N=162$ they decrease up to the local minimum at $N=170$. The drop is very steep for the lighter elements (Rf, Sg, and $\mathrm{Hs}$ ) and gentler for the heavier ones (Ds and $\mathrm{Cn}$ ), whereas for the isotopes of $Z=114$ almost no change is observed. This behavior can be explained by the decreasing potential energy along almost the whole barrier, associated with a diminishing of the saddle point energy and the clear development of the second minimum (Figs. 9, 10, and 11). The inverse trend of increasing the potential energy at small deformations is noticed in heavier elements, starting from $\mathrm{Cn}$ (Fig. 11). The first RS hump of the barrier grows with increasing mass of the nucleus. In the heavy isotopes with $A \geqslant 280$ it is higher than the second reflection-symmetric barrier.

The half-lives calculated for $162 \leqslant N \leqslant 170$ along the non-reflection-symmetric path diminish more rapidly with the increase of $N$ than in the reflection-symmetric mode. This is induced by the changes that take place in the nonreflection-symmetric barrier, namely, the fast decrease of its height and, more important, the narrowing of its width (see Figs. 10 and 11). In nuclei with $N \geqslant 170$ the nonreflection-symmetric fission barrier allows the second hump of the reflection-symmetric barrier to be avoided, making asymmetric fission the most probable mode. Very short fission half-lives, even below $T=1 \mathrm{~ms}$, can be found in the Hs, Ds, $\mathrm{Cn}$, and $Z=114$ isotopes, where non-reflection-symmetric fission is the dominant decay mode, being even faster than $\alpha$ emission. In $Z=114$ non-axially-symmetric fission has half-lives comparable to that of $\alpha$ decay.

The available experimental data around $N=172$ obtained from the "hot fusion" experiments [8,9] fit the theoretical predictions perfectly. In $\mathrm{Cn}$ two spontaneous fission half-lives correspond to the prediction of the non-reflection-symmetric fission mode. In $Z=114, N=172$ the observed $50 \%$ branching ratio is very well reproduced. In the isotope with two more neutrons the detected $\alpha$ decay is predicted with only slightly longer half-life than in the dominant fission channel. Finally, in $Z=116$ and $Z=118$ the dominant $\alpha$ radioactivity is properly predicted by the theoretical analysis with a good estimation of the half-lives.

While approaching $N=178$ the fission half-lives increase with a slope which grows with the proton number. This effect is governed by two trends observed in the evolution of the PESs. The first one is the aforementioned growth of the first hump of the barrier, which is the highest one in almost all nuclei with $N>170$. The other factor is the lowering of the energy of the oblate minimum, which becomes the ground state in the nuclei around $N=178$ (see also the discussions of groundstate deformations in Sec. III). The shift of the ground state from the prolate to the oblate minimum (see also Fig. 2) gives 
an additional increase of the barrier height up to over $1 \mathrm{MeV}$. The energy difference between prolate and oblate minima has its largest value in the $N=178$ isotones, which, when added to the large first barrier, produces extremely long half-lives. They exceed (in the non-reflection-symmetric mode) $T=10^{7}$ $\mathrm{s}$ in $\mathrm{Cn}$ and $Z=114$ and $T=10^{14} \mathrm{~s}$ in $Z=120$.

In the neutron-rich $\mathrm{Hs}$ isotopes the non-reflectionsymmetric barriers extend up to relatively large values of the quadrupole moment. Therefore their transition probabilities are smaller than in the reflection-symmetric mode. This fact together with the long $\alpha$ decay half-lives implies that in this region one can find isotopes with very long half-lives. Here reflection-symmetric fission is the dominant decay mode. However, these very neutron rich Hs isotopes are extremely difficult to synthesize using contemporary experimental techniques.

\section{The region $N \geqslant 180$}

The saddle point of the first hump of the barrier takes its maximal energy value at $N=182$. We can observe in these isotones another very high maximum of the fission half-lives in all elements. In contrast, the $N=180$ isotones decay a few orders of magnitude faster than the neighboring nuclei with $N=178$ and $N=182$. The influence of the oblate minimum and the high first barrier is not strong enough to enlarge the fission half-lives here. In two isotones with $N=180$, namely, $\mathrm{Cn}$ and $Z=114$, non-reflection-symmetric fission is the dominant process with half-lives shorter than for $\alpha$ decay.

The energy difference between the oblate and the prolate minima as well as between the absolute values of their quadrupole moments shrink in $N=180$ and $N=182$ continuously. Finally, one finds that all $N=184$ isotones have a spherical ground state. This indicates a magic number at $N=184$. Nevertheless, this feature does not have a big impact on the fission half-lives and the region of the most stable nuclei is slightly shifted toward the neutron-deficient isotopes.

At $N=178$ and $N=182$ the $\alpha$-decay half-lives also reach their maxima although they are less pronounced than for the fission half-lives. They correspond to the minima of $Q_{\alpha}$ that can be observed for these neutron numbers (see Fig. 5). For the $Z \geqslant 116$ elements $\alpha$ emission is the fastest decay process for isotopes lighter than $N=184$. Most of them live long enough to allow the synthesis of these nuclei. The extremely long fission half-lives for the $N=178$ and $N=182$ isotopes are larger than the $\alpha$-decay half-lives in $\mathrm{Cn}$ and $Z=114$ whereas for $N=180$ non-reflection-symmetric fission is the dominant mode.

The isotopes with neutron number larger than the magic $N=184$ are characterized by a rapid decrease of the fission half-lives with increasing neutron number. This is a consequence of the decrease of energy along the whole energy barrier. The second minimum goes down below the ground state and the height of the first barrier reduces substantially when heavier nuclei are considered. These strong trends cannot be balanced by the few-MeV decrease of the ground-state energy due to the octupole deformation. Consequently, for the elements from Ds to $Z=120$, fission becomes the dominant decay mode with half-lives below $T=10 \mu \mathrm{s}$. The $\alpha$-decay

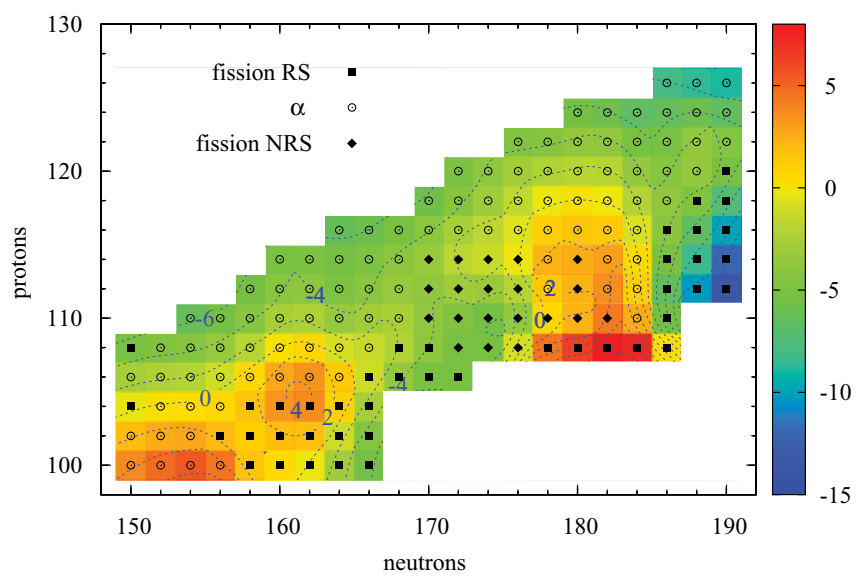

FIG. 15. (Color online) The dominant decay modes of SHEs. The logarithm of the shortest half-lives (in seconds) is also indicated.

half-lives do not vary strongly along the isotopic chain in this region. The fluctuations are associated with the changes of the ground-state deformations of the parent and the daughter nuclei. In this region the decrease of the $\alpha$-decay half-lives with the proton number is also visible. Most isotopes in this region cannot be synthesized due to the very short fission (in the proton-deficient nuclei) or $\alpha$ emission (in the proton-rich isotones) half-lives. The experimental limit of $T=1 \mathrm{~ms}$ is exceeded only for a few nuclei.

To conclude this section we would like to present in Fig. 15 the shortest half-lives of each isotope in the form of the chart of SHEs. From this figure it is easy to distinguish the regions where each decay mode plays the most important role. The predominant decay mode, especially in the proton-rich region, is $\alpha$ emission. Roughly speaking, the spontaneous fission in the reflection-symmetric mode is dominant in the proton-deficient nuclei with $Z \leqslant 104$ for $N \geqslant 158, Z \leqslant 108$ for $N \geqslant 170$, and $Z \leqslant 116$ for $N \geqslant 186$. In the central part of the diagram the region with the fastest decay in the non-reflection-symmetric fission channel is defined by $108 \leqslant Z \leqslant 114$ and $170 \leqslant N \leqslant$ 180.

Two regions of long-living nuclei can be found also in Fig. 15. The first one includes nuclei around ${ }^{268} \mathrm{Sg}_{162}$, where half-lives reach $T=10^{4} \mathrm{~s}$. The other "island of stability" is associated with the anomalous long fission half-lives at $N=$ 178 and $N=182$. At these neutron numbers (and also at $N=184$ ) long-living isotopes of $\mathrm{Cn}$ and $Z=114$ elements can be found. The half-lives of these isotopes are also longer than $T=10^{6} \mathrm{~s}$. Very long half-lives characterize also the Hs isotopes decaying through reflection-symmetric fission.

\section{SUMMARY AND CONCLUSIONS}

In this work Hatree-Fock-Bogoliubov theory with large basis size and the density-dependent Gogny force as interaction have been used to study the most relevant properties of 160 heavy and superheavy elements as well as their predominant decay modes. In order to keep the calculations as general as possible we allow for wave functions with different symmetries, namely, axially symmetric and reflection 
symmetric, axially symmetric and non-reflection symmetric, and lastly triaxial wave functions. After a thorough analysis along different fission paths and considering that our calculations are extremely demanding of computation time due to the large basis needed to study 160 nuclei we perform most calculations in the axially symmetric approaches.

The ground-state deformations $\beta_{2}, \beta_{3}, \beta_{4}$, and $\beta_{6}$ as well as $Q_{\alpha}$ factors, pairing properties, and the two-neutron and two proton-separation energies are thoroughly discussed. The single-particle energies are used as a guide for the interpretation of these properties.

The fission paths for three representative nuclei, namely, ${ }^{274} \mathrm{Hs},{ }^{278} \mathrm{Ds}$, and ${ }^{282} \mathrm{Cn}$, are analyzed in detail. Properties such as mass parameters, pairing features, and the variation of the action among others are calculated along the fission path with wave functions of the three types mentioned above. We find that though the shape of the fission barrier has a large impact on the fission half-lives, the mass parameter also plays an important role, in such a way that wave functions with larger barriers and smaller mass parameter may tunnel more easily than alternative ones with smaller barriers and larger mass. Since in general axially symmetric wave functions do have smaller masses than the triaxial ones, the restriction to axial symmetry is a good option for performing a systematic study of the half-lives of SHEs. In the second part of the paper a thorough study of the shapes of the barriers in the AS-RS and AS-NRS cases is performed. A reasonable explanation of heights and shapes as well as of octupole effects is obtained on the basis of the single-particle energy levels. The twodimensional $\left(Q_{2}, Q_{3}\right)$ energy contour plots for the nuclei ${ }^{274} \mathrm{Hs}$ and ${ }^{282} \mathrm{Cn}$ allow us to disentangle the different fission paths and their possible interconnections.

In the third part of the paper the half-lives of all studied nuclei are calculated for the different decay modes, namely, $\alpha$ decay and along the different fission paths. We find clear tendencies with the neutron number that are easily explainable on the basis of the behavior of $Q_{\alpha}$ factors and barrier shapes, respectively. In particular, we find that $\alpha$ emission is the predominant decay mode, especially in the proton-rich region. For spontaneous fission we obtain that the reflectionsymmetric mode is dominant for the proton-deficient nuclei in medium-mass SHEs with $Z \leqslant 104$ for $N \geqslant 158$ and with $Z \leqslant 108$ for $N \geqslant 170$ and in the region of the heaviest SHEs with $Z \leqslant 116$ for $N \geqslant 186$. The fastest decay in the non-reflection-symmetric fission channel takes place for $108 \leqslant Z \leqslant 114$ and $170 \leqslant N \leqslant 180$. The long-living nuclei can be found in two regions. The first one is in the vicinity of ${ }^{268} \mathrm{Sg}_{162}$, where half-lives around $T=10^{4} \mathrm{~s}$ are obtained. The anomalous long fission half-lives for proton-deficient isotones with $N=178, N=182$, and $N=184$ create the second region. At these neutron numbers several isotopes of the elements $\mathrm{Cn}$ and $Z=114$ are found with half-lives longer than $T=10^{6} \mathrm{~s}$. Very long half-lives characterize also the Hs isotopes decaying through reflection-symmetric fission. The nuclei beyond $Z=120$ and $N=184$ have half-lives too short to be detected within the contemporary experimental limit of $T=10 \mu \mathrm{s}$.

In conclusion, we have presented a systematic study of SHEs within self-consistent HFB theory with very general wave functions and large configuration space. Our calculations provide an overall interpretation of the systematics and global properties of these elements and its decay modes. In general we find a reasonably good agreement with the experimental data.

\section{ACKNOWLEDGMENTS}

The authors would like to thank Prof. Krzysztof Pomorski for a careful reading of the manuscript. The contribution of Luis M. Robledo to this paper is gratefully acknowledged. This work partially supported by Grant No. DEC-2011/01/B/ST2/03667 from the National Science Centre (Poland) and by the Spanish Ministerio de Educación y Ciencia under Contracts No. FPA2009-13377-C02-01 and No. FPA2011-29854-C04-04 and the Spanish ConsoliderIngenio 2010 Programme CPAN (CSD2007-00042).
[1] D. C. Hoffman, Nucl. Phys. A 502, 21c (1989).

[2] F. Gönnenwein, Nucl. Phys. A 654, 855c (1999).

[3] S. Hofmann et al., Eur. Phys. J. A 32, 251 (2007).

[4] F. P. Heßberger et al., Eur. Phys. J. A 41, 145 (2009).

[5] Ch. E. Düllmann et al., Phys. Rev. Lett. 104, 252701 (2010).

[6] F. P. Heßberger, S. Antalic, D. Ackermann, S. Heinz, S. Hofmann, J. Khuyagbaatar, B. Kindler, I. Kojouharov, B. Lommel, and R. Mann, Eur. Phys. J. A 43, 175 (2010).

[7] J. Khuyagbaatar et al., Eur. Phys. J. A 46, 59 (2010).

[8] J. M. Gates et al., Phys. Rev. C 83, 054618 (2011).

[9] Yu. Ts. Oganessian et al., Phys. Rev. C 72, 034611 (2005).

[10] Yu. Ts. Oganessian, J. Phys. G 34, R165 (2007).

[11] Yu. Ts. Oganessian et al., Phys. Rev. C 76, 011601(R) (2007).

[12] Yu. Ts. Oganessian et al., Phys. Rev. C 79, 024603 (2009).

[13] Yu. Ts. Oganessian et al., Phys. Rev. Lett. 104, 142502 (2010).

[14] Yu. Ts. Oganessian et al., Phys. Rev. Lett. 108, 022502 (2012).

[15] K. Morita et al., J. Phys. Soc. Jpn. 73, 2593 (2004).
[16] K. Morita et al., J. Phys. Soc. Jpn. 76, 045001 (2007).

[17] K. Morita et al., J. Phys. Soc. Jpn. 78, 064201 (2009).

[18] H. Haba et al., Phys. Rev. C 83, 034602 (2011).

[19] Z. Sosin et al., Acta Phys. Pol. B 40, 741 (2009).

[20] J. Gerl, Acta Phys. Pol. B 40, 767 (2009).

[21] L. Stavsetra, K. E. Gregorich, J. Dvorak, P. A. Ellison, I. Dragojević, M. A. Garcia, and H. Nitsche, Phys. Rev. Lett. 103, 132502 (2009).

[22] K. Moody (Dubna-Livermore Collaboration), Nucl. Phys. A 734, 188 (2004).

[23] Ch. Theisen, Acta Phys. Pol. B 38, 1525 (2007).

[24] R. Julin, Nucl. Phys. A 834, 15c (2010).

[25] A. Sobiczewski and K. Pomorski, Prog. Part. Nucl. Phys. 58, 292 (2007).

[26] A. Sobiczewski, F. A. Gareev, and B. N. Kalinkin, Phys. Lett. 22, 500 (1966).

[27] W. D. Myers and W. J. Świątecki, Nucl. Phys. 81, 1 (1966).

[28] U. Mosel and W. Greiner, Z. Phys. 222, 261 (1969). 
[29] A. Baran, K. Pomorski, A. Łukasiak, and A. Sobiczewski, Nucl. Phys. A 361, 83 (1981).

[30] Z. Łojewski and A. Baran, Z. Phys. A 322, 695 (1985).

[31] A. Staszczak and Z. Łojewski, Nucl. Phys. A 657, 134 (1999).

[32] I. Muntian, S. Hofmann, Z. Patyk, and A. Sobiczewski, Acta Phys. Pol. B 34, 2073 (2003).

[33] M. Kowal, P. Jachimowicz, and A. Sobiczewski, Phys. Rev. C 82, 014303 (2010)

[34] P. Möller, J. R. Nix, and W. J. Świątecki, Nucl. Phys. A 469, 1 (1987).

[35] P. Möller, J. R. Nix, and W. J. Świątecki, Nucl. Phys. A 492, 349 (1989).

[36] P. Möller and A. Iwamoto, Phys. Rev. C 61, 047602 (2000).

[37] P. Möller, D. G. Madland, A. J. Sierk, and A. Iwamoto, Nature 409, 785 (2001).

[38] P. Möller and J. R. Nix, Nucl. Phys. A 549, 84 (1992).

[39] Y. K. Gambhir, A. Bhagwat, and M. Gupta, Ann. Phys. (NY) 320, 429 (2005).

[40] S. Kumar, Phys. Rev. C 85, 024320 (2012).

[41] T. Bürvenich, M. Bender, J. A. Maruhn, and P.-G. Reinhard, Phys. Rev. C 69, 014307 (2004).

[42] M. Bender, K. Rutz, P.-G. Reinhard, J. A. Maruhn, and W. Greiner, Phys. Rev. C 58, 2126 (1998).

[43] S. Karatzikos, A. V. Afanasjev, G. A. Lalazissis, and P. Ring, Phys. Lett. B 689, 72 (2010).

[44] H. Abusara, A. V. Afanasjev, and P. Ring, Phys. Rev. C 82, 044303 (2010)

[45] H. Abusara, A. V. Afanasjev, and P. Ring, Phys. Rev. C 85, 024314 (2012).

[46] B.-N. Lu, E.-G. Zhao, and S.-G. Zhou, Phys. Rev. C 85, 011301(R) (2012).

[47] H. Flocard, in Nuclear Self-Consistent Fields, edited by G. Ripka and M. Porneuf (North-Holland, New York, 1975), p. 219.

[48] S. Ćwiok, J. Dobaczewski, P.-H. Heenen, P. Magierski, and W. Nazarewicz, Nucl. Phys. A 611, 211 (1996).

[49] K. Rutz, M. Bender, T. Bürvenich, T. Schilling, P.-G. Reinhard, J. A. Maruhn, and W. Greiner, Phys. Rev. C 56, 238 (1997).

[50] M. Bender, K. Rutz, P.-G. Reinhard, J. A. Maruhn, and W. Greiner, Phys. Rev. C 60, 034304 (1999).

[51] A. Staszczak, J. Dobaczewski, and W. Nazarewicz, Int. J. Mod. Phys. E 14, 395 (2005)

[52] J. F. Berger and K. Pomorski, Phys. Rev. Lett. 85, 30 (2000).

[53] J.-F. Berger, L. Bitaud, J. Dechargé, M. Girod, and K. Dietrich, Nucl. Phys. A 685, 1c (2001).

[54] M. Warda, J. L. Egido, and L. M. Robledo, Int. J. Mod. Phys. E 15, 504 (2006)

[55] F. A. Ivanyuk and K. Pomorski, Phys. Rev. C 79, 054327 (2009).

[56] A. Staszczak, S. Piłat, and K. Pomorski, Nucl. Phys. A 504, 589 (1989).

[57] M. Warda, J. L. Egido, L. M. Robledo, and K. Pomorski, Phys. Rev. C 66, 014310 (2002).

[58] V. Y. Denisov and A. A. Khudenko, Phys. Rev. C 81, 034613 (2010).

[59] N. Dasgupta-Schubert, M. A. Reyes, and V. A. Tamez, Eur. Phys. J. A 42, 121 (2009).

[60] [http://www.iupac.org/web/nt/2010-02-20/112/Copernicium].

[61] J. F. Berger, M. Girod, and D. Gogny, Nucl. Phys. A 428, 23c (1984).
[62] J. F. Berger, M. Girod, and D. Gogny, Comput. Phys. Commun. 63, 365 (1991).

[63] J. Dechargé and D. Gogny, Phys. Rev. C 21, 1568 (1980).

[64] J. F. Berger, M. Girod, and D. Gogny, Nucl. Phys. A 502, 85c (1989).

[65] J. F. Berger, L. Bitaud, J. Dechargé, M. Girod, and S. Peru-Dessenfants, in Proceedings of the International Workshop XXXIV on Gross Properties of Nuclei and Nuclear Excitations, Hirschegg, Austria, 1996, edited by H. Feldmeier, J. Knoll, and W. Nörenberg (GSI, Darmstadt, 1996), p. 56.

[66] M. Girod and B. Grammaticos, Phys. Rev. C 27, 2317 (1983).

[67] J. L. Egido and L. M. Robledo, Nucl. Phys. A 494, 85 (1989).

[68] M. Girod, J. P. Delaroche, D. Gogny, and J. F. Berger, Phys. Rev. Lett. 62, 2452 (1989).

[69] J. L. Egido and L. M. Robledo, Phys. Rev. Lett. 70, 2876 (1993).

[70] J. P. Blaizot, J. F. Berger, J. Dechargé, and M. Girod, Nucl. Phys. A 591, 435 (1995).

[71] E. Garrote, J. L. Egido, and L. M. Robledo, Phys. Rev. Lett. 80, 4398 (1998).

[72] R. Rodríguez-Guzmán, J. L. Egido, and L. M. Robledo, Nucl. Phys. A 709, 201 (2002).

[73] M. Warda, K. Pomorski, J. L. Egido, and L. M. Robledo, Int. J. Mod. Phys. E 14, 403 (2005).

[74] M. Warda, K. Pomorski, J. L. Egido, and L. M. Robledo, J. Phys. G 31, S1555 (2005).

[75] M. Warda, J. L. Egido, and L. M. Robledo, Phys. Scr. T125, 226 (2006).

[76] T. R. Rodríguez and J. L. Egido, Phys. Rev. Lett. 99, 062501 (2007).

[77] T. R. Rodríguez and J. L. Egido, Phys. Rev. C 81, 064323 (2010).

[78] N. López Vaquero, T. R. Rodríguez, and J. L. Egido, Phys. Lett. B 704, 520 (2011).

[79] M. Warda and L. M. Robledo, Phys. Rev. C 84, 044608 (2011).

[80] J. L. Egido, L. M. Robledo, and R. R. Chasman, Phys. Lett. B 393, 13 (1997).

[81] C. Titin-Schnaider and Ph. Quentin, Phys. Lett. B 49, 213 (1974).

[82] M. Anguiano, J. L. Egido, and L. M. Robledo, Nucl. Phys. A 683, 227 (2001).

[83] J. L. Egido and L. M. Robledo, Angular Momentum Projection and Quadrupole Correlations Effects in Atomic Nuclei, Lecture Notes in Physics Vol. 641 (Springer, New York, 2004) p. 269.

[84] R. R. Rodríguez-Guzmán, J. L. Egido, and L. M. Robledo, Phys. Rev. C 62, 054319 (2000); Phys. Lett. B 474, 15 (2000); Acta Phys. Pol. B 32, 2385 (2001).

[85] K. Dietrich, H. J. Mang, and J. H. Pradal, Phys. Rev. 135, B22 (1964).

[86] P. Ring and P. Schuck, The Nuclear Many-Body Problem (Springer-Verlag, Berlin, 1980).

[87] J. L. Egido and L. M. Robledo, Phys. Rev. Lett. 85, 1198 (2000).

[88] M. Girod, J. P. Delaroche, J. Libert, and I. Deloncle, Phys. Rev. C 45, R1420 (1992).

[89] D. L. Hill and J. A. Wheeler, Phys. Rev. 89, 1102 (1953).

[90] M. J. Giannoni and Ph. Quentin, Phys. Rev. C 21, 2060 (1980)

[91] S. G. Nilsson, C. F. Tsang, A. Sobiczewski, Z. Szymański, S. Wycech, Ch. Gustafson, I.-L. Lamm, P. Möller, and B. Nilsson, Nucl. Phys. A 131, 1 (1969). 
[92] S. Ćwiok, P.-H. Heenen, and W. Nazarewicz, Nature (London) 433, 705 (2005).

[93] V. E. Viola Jr. and G. T. Seaborg, J. Inorg. Nucl. Chem. 28, 741 (1966).

[94] G. Audi, A. H. Wapstra, and C. Thibault, Nucl. Phys. A 729, 337 (2003).

[95] Z. Patyk and A. Sobiczewski, Nucl. Phys. A 533, 132 (1991).

[96] P. Jachimowicz, M. Kowal, and J. Skalski, Phys. Rev. C 83, 054302 (2011).

[97] J. F. Berger, D. Hitara, M. Girod, and J. Dechargé, Int. J. Mod. Phys. E 13, 79 (2004).

[98] J.-F. Berger, L. Bitaud, J. Decharge, M. Girod, and K. Dietrich, Nucl. Phys. A 685, 1c (2001).

[99] P. Möller, J. R. Nix, W. D. Myers, and W. J. Świątecki, At. Data Nucl. Data Tables 59, 185 (1995).
[100] A. Sobiczewski and I. Muntian, Int. J. Mod. Phys. E 14, 409 (2005).

[101] A. Baran, Z. Łojewski, K. Sieja, and M. Kowal, Phys. Rev. C 72, 044310 (2005).

[102] S. Goriely, J. M. Pearson, and F. Tondeur, Nucl. Phys. A 688, 349c (2001).

[103] F. Chappert, M. Girod, and S. Hilaire, Phys. Lett. B> 668, 420 (2008).

[104] S. Goriely, S. Hilaire, M. Girod, and S. Péru, Phys. Rev. Lett. 102, 242501 (2009).

[105] W. Nazarewicz and S. L. Tabor, Phys. Rev. C 45, 2226 (1992).

[106] D. N. Poenaru, R. A. Gherghescu, and W. Greiner, Phys. Rev. C 85, 034615 (2012).

[107] G. Audi, O. Bersillon, J. Blachot, and A. H. Wapstra, Nucl. Phys. A 729, 3 (2003). 